TRANSACTIONS OF THE

AMERICAN MATHEMATICAL SOCIETY

Volume 357, Number 6, Pages 2205-2233

S 0002-9947(05)03847-X

Article electronically published on January 31, 2005

\title{
ORBIFOLDS AND ANALYTIC TORSIONS
}

\author{
XIAONAN MA
}

\begin{abstract}
In this paper, we calculate the behavior of the Quillen metric by orbifold immersions. We thus extend a formula of Bismut-Lebeau to the orbifold case.

RÉSumÉ. Dans cet article, on calcule le comportement de métrique de Quillen par immersions d'orbifold. On étend ainsi une formule de Bismut-Lebeau au cas d'orbifold.
\end{abstract}

\section{INTRODUCTION}

Let $\xi$ be a Hermitian vector bundle on a compact Hermitian complex manifold $X$. Let $\lambda(\xi)=\bigotimes_{i}\left(\operatorname{det} H^{i}(X, \xi)\right)^{(-1)^{i+1}}$ be the inverse of the determinant of the cohomology of $\xi$. Quillen 28] first defined a metric on $\lambda(\xi)$ in the case where $X$ is a Riemann surface. Quillen metric is the product of the $L_{2}$ metric on $\lambda(\xi)$ by the Ray-Singer analytic torsion of the Dolbeault complex. The logarithm of the RaySinger analytic torsion 29 is a linear combination of derivatives at zero of the zeta function of the Hodge Laplacians acting on smooth forms of various degrees. In [10], Bismut, Gillet, and Soulé have extended it to complex manifolds. They have established the anomaly formulas for Quillen metrics, which tell us the variation of Quillen metrics on the metrics on $\xi$ and $T X$ by using some Bott-Chern classes. Later, Bismut and Köhler [12] (cf. also [9], 18]) have extended the analytic torsion of Ray-Singer to the analytic torsion forms $T$ for a holomorphic submersion. In particular, the equation on $\frac{\bar{\partial} \partial}{2 i \pi} T$ gives a refinement of the Grothendieck-RiemannRoch Theorem to the level of differential forms. They have also established the corresponding anomaly formulas.

Let $i: Y \rightarrow X$ be an immersion of compact complex manifolds. Let $\eta$ be a holomorphic vector bundle on $Y$, and let $(\xi, v)$ be a complex of holomorphic vector bundles which provides a resolution of $i_{*} \eta$. Then by [23], the line $\lambda^{-1}(\eta) \otimes \lambda(\xi)$ has a nonzero canonical section $\sigma$. In [13], Bismut and Lebeau have given a formula for the Quillen norm of $\sigma$ in terms of Bott-Chern currents on $X$ and of a genus $R$ introduced by Gillet and Soulé [18]. In [19], the result of 13] was used by Gillet and Soulé to obtain an arithmetic Riemann-Roch Theorem in Arakelov geometry. More recently, in 24] and their later works, Köhler and Roessler proved a Lefschetz fixed point formula in Arakelov geometry, and the results on the equivariant analytic torsion (forms) in [7], 14] play also an important role in their proof.

In this paper, we extend some results on analytic torsions to the orbifold case. Historically, orbifold first appeared as $V$-manifolds in Satake's extension [30] of

Received by the editors July 10, 2003.

2000 Mathematics Subject Classification. Primary 57J52, 32L10, 58J20. 
the Gauss-Bonnet-Chern Theorem in 1957 and in Kawasaki's extension [21] of the Riemann-Roch Theorem in 1978. It turns out that orbifolds appear naturally in mathematics and physics; for example, the symplectic reduction, the problems on moduli spaces, and the orbifold string theory. In recent years, orbifolds became a popular subject. We can find many interesting results and various aspects on orbifolds in the recent work [1]. The Kawasaki-Riemann-Roch Theorem has also found many applications, thus it is a natural pursuit to understand "secondary" spectral invariants such as analytic torsions and $\eta$-invariants on orbifolds.

We will use the heat kernel methods to solve our problem. Thanks to finite propagation speed of solutions of hyperbolic equations, we can localize the problem. Since, locally, we have to meet $G$-manifolds, to generalize the results to the orbifold case, we must well understand the situations of $G$-equivariant complex manifold cases which were done in [6] and [27. After localized, we can apply the techniques of [6] and 27] to our case. We also hope our results have corresponding versions in Arakelov geometry. In this direction, we establish the analytic part of "arithmetic Kawasaki-Riemann-Roch Theorem".

Now we explain our results in more detail. Let $\xi$ be a holomorphic orbifold vector bundle on a complex orbifold $X$. Let $\Sigma X$ be the strata of $X$ which has a natural orbifold structure. Let $m_{i}$ be the multiplicity of connected component $X_{i}$ of $X \cup \Sigma X$ (cf. (1.2)).

In all the following formulas, we must consider the differential forms on $X \cup \Sigma X$, not on $X$. For example, $\operatorname{Td}^{\Sigma}\left(T X, g^{T X}\right)$ is the Chern-Weil Todd form on $X \cup \Sigma X$ associated to the holomorphic Hermitian connection on $\left(T X, g^{T X}\right)$, which appears in Kawasaki's formulas [21. Other Chern-Weil forms will be denoted in a similar way. In particular, the forms $\mathrm{ch}^{\Sigma}\left(\xi, h^{\xi}\right)$ on $X \cup \Sigma X$ are the Chern character forms of the maximum proper orbifold sub-bundle of $\left(\xi, h^{\xi}\right)$ (cf. Section 1.2).

At first, we establish the anomaly formula for Quillen metrics on $\lambda(\xi)$. Let $g^{\prime T X}, h^{\prime} \xi$ be another couple of metrics on $T X, \xi$. Let \|\|$_{\lambda(\xi)}$ (resp. \|\|$_{\lambda(\xi)}^{\prime}$ ) be the Quillen metric on $\lambda(\xi)$ associated to $g^{T X}, h^{\xi}$ (resp. $g^{T X}, h^{\prime \xi}$ ). In Section 1.2, we construct the Bott-Chern classes $\widetilde{\mathrm{Td}}^{\Sigma}\left(T X, g^{T X}, g^{\prime T X}\right)$ and $\widetilde{\mathrm{ch}^{\Sigma}}\left(\xi, h^{\xi}, h^{\prime \xi}\right)$ on $X \cup \Sigma X$.

The following result extends the anomaly formulas of [10, Theorem 1.23] to orbifolds.

Theorem 0.1. Assume that the metrics $g^{T X}$ and $g^{\prime T X}$ are Kähler. Then

$$
\begin{aligned}
\log \left(\frac{\|\|_{\lambda(\xi)}^{\prime 2}}{\|\|_{\lambda(\xi)}^{2}}\right)=\sum_{i} & \left(\frac{1}{m_{i}} \int_{X_{i}} \widetilde{\operatorname{Td}}^{\Sigma}\left(T X, g^{T X}, g^{\prime T X}\right) \operatorname{ch}^{\Sigma}\left(\xi, h^{\xi}\right)\right. \\
& \left.+\frac{1}{m_{i}} \int_{X_{i}} \operatorname{Td}^{\Sigma}\left(T X, g^{\prime T X}\right){\widetilde{\mathrm{ch}^{\Sigma}}}^{\Sigma}\left(\xi, h^{\xi}, h^{\prime \xi}\right)\right) .
\end{aligned}
$$

Let $i: Y \rightarrow X$ be an orbifold embedding of compact complex orbifolds. (Locally, if $\pi: U \rightarrow V$ is a $G$-equivariant holomorphic map of complex manifolds with a finite group $G$, then we say $\pi: U / G \rightarrow V / G$ is an immersion if $\pi: U \rightarrow V$ is an immersion.) Let $\eta$ be a holomorphic orbifold vector bundle on $Y$ and let

$$
(\xi, v): 0 \rightarrow \xi_{m} \rightarrow \cdots \rightarrow \xi_{0} \rightarrow 0
$$

be a holomorphic chain complex of orbifold vector bundles on $X$ which, together with a restriction map, $r:\left.\xi_{0}\right|_{Y} \rightarrow \eta$, provides a orbifold resolution of the sheaf 
$i_{*} \mathcal{O}_{Y}(\eta)$. Set $\lambda(\xi)=\bigotimes_{i=0}^{m} \lambda\left(\xi_{i}\right)^{(-1)^{i}}$. Let $\sigma$ be the canonical nonzero element of the line $\lambda^{-1}(\eta) \otimes \lambda(\xi)$.

Assume that $T X, T Y, \xi_{0}, \cdots, \xi_{m}, \eta$ are equipped with Hermitian metrics. Our first assumption is that the metric $g^{T X}$ on $T X$ is Kähler and that the metric $g^{T Y}$ on $T Y$ is induced by the metric $g^{T X}$. Let $N$ be the normal orbifold bundle to $Y$ in $X$, and let $g^{N}$ be the metric induced by $g^{T X}$ on $N$. We assume in addition that assumption (A) in Bismut [3, Definition 1.5], is verified. i.e. that the metric $h^{\xi_{0}}, \cdots, h^{\xi_{m}}$ on $\xi_{0}, \cdots, \xi_{m}$ are in some sense compatible with the metrics $g^{N}, h^{\eta}$ on $N, \eta$.

In Section 4, we construct a sub-orbifold $Y^{\prime}$ of $\Sigma X$ from the immersion $i: Y \rightarrow$ $X$. Remark that, in general, $Y^{\prime} \neq \Sigma Y$, and $\Sigma Y$ is not a sub-orbifold of $\Sigma X$. Let $T^{\Sigma}\left(\xi, h^{\xi}\right)$ be the Bott-Chern current on $X \cup \Sigma X$ constructed in (4.4), associated to the complex $(\xi, v)$, such that

$$
\frac{\bar{\partial} \partial}{2 i \pi} T^{\Sigma}\left(\xi, h^{\xi}\right)=\left(\mathrm{Td}^{\Sigma}\right)^{-1}\left(N, g^{N}\right) \operatorname{ch}^{\Sigma}\left(\eta, h^{\eta}\right) \delta_{Y \cup Y^{\prime}}-\operatorname{ch}^{\Sigma}\left(\xi, h^{\xi}\right) .
$$

Let $\widetilde{\mathrm{Td}}^{\Sigma}\left(T Y,\left.T X\right|_{Y}, g^{T X}\right)$ be the Bott-Chern class on $Y \cup Y^{\prime}$ constructed as in [8, §1f)], associated to the complex $\left.0 \rightarrow T Y \rightarrow T X\right|_{Y} \rightarrow N \rightarrow 0$, such that

$$
\frac{\bar{\partial} \partial}{2 i \pi} \widetilde{\mathrm{Td}}^{\Sigma}\left(T Y,\left.T X\right|_{Y}, g^{T X}\right)=\operatorname{Td}^{\Sigma}\left(T X, g^{T X}\right)-\operatorname{Td}^{\Sigma}\left(T Y, g^{T Y}\right) \operatorname{Td}^{\Sigma}\left(N, g^{N}\right) .
$$

Finally, let $R(\theta, x)$ be the power series introduced by [5]. We identify $R^{\Sigma}$ with the corresponding additive genus as in (5.8).

Let $m_{i, X}$ be the multiplicity of connected components $X_{i}$ of $X \cup \Sigma X$. For any connected component $Y_{j}^{\prime}$ of $Y \cup Y^{\prime}$, let $m_{X_{i}, Y_{j}^{\prime}}$ be the relative multiplicity of $Y_{j}^{\prime} \subset X_{i}$ (cf. Definition 1.6). In Section 6, we establish the following extension of [13, Theorem 0.1].

Theorem 0.2. The following identity holds:

$$
\begin{aligned}
& \log \left(\|\sigma\|_{\lambda^{-1}(\eta) \otimes \lambda(\xi)}^{2}\right)=\sum_{i} \frac{1}{m_{i, X}}\left[-\int_{X_{i}} \operatorname{Td}^{\Sigma}\left(T X, g^{T X}\right) T^{\Sigma}\left(\xi, h^{\xi}\right)\right. \\
& \quad+\sum_{j} \frac{1}{m_{X_{i}, Y_{j}^{\prime}}} \int_{Y_{j}^{\prime}}\left(\mathrm{Td}^{-1}\right)^{\Sigma}\left(N, g^{N}\right) \widetilde{\mathrm{Td}}^{\Sigma}\left(\left.T Y\right|_{Y_{j}^{\prime}},\left.T X\right|_{Y_{j}^{\prime}}, g^{T X}\right) \operatorname{ch}^{\Sigma}\left(\eta, h^{\eta}\right) \\
& \left.\quad-\sum_{j} \frac{1}{m_{X_{i}, Y_{j}^{\prime}}} \int_{Y_{j}^{\prime}} \operatorname{Td}^{\Sigma}(T X) R^{\Sigma}(N) \operatorname{ch}^{\Sigma}(\eta)\right] .
\end{aligned}
$$

Needless to say, Theorems 0.10 .2 are direct consequences of $[6$, Theorems 2.5, 0.1 for quotients of manifolds by finite group actions. More precisely, let $G$ be a finite group and let $\widetilde{\xi}$ be a holomorphic vector bundle on a compact complex manifold $\widetilde{X}$. If $G$ acts holomorphically on $\widetilde{X}$ and its action lifts on $\widetilde{\xi}$, then $\xi=\widetilde{\xi} / G$ is an orbifold vector bundle on $X=\widetilde{X} / G$. Let $G_{1}=\operatorname{Ker}\{G \rightarrow \operatorname{Diffeo}(\widetilde{X})\}$. Then the group $G / G_{1}$ acts effectively on $\widetilde{X}$ and the $G_{1}$-invariant part of $\widetilde{\xi}$ forms a vector bundle $\widetilde{\xi}^{G_{1}}$ on $\widetilde{X}$. Now $\mathcal{C}^{\infty}\left(X, \Lambda\left(T^{*(0,1)} X\right) \otimes \xi\right)$ is the $G$-invariant $\mathcal{C}^{\infty}$ sections of $\Lambda\left(T^{*(0,1)} \widetilde{X}\right) \otimes \widetilde{\xi}$ on $\widetilde{X}$, i.e. the $G / G_{1}$-invariant $\mathcal{C}^{\infty}$ sections of $\Lambda\left(T^{*(0,1)} \widetilde{X}\right) \otimes \widetilde{\xi}^{G_{1}}$ 
on $\tilde{X}$. Thus by [6] (2.11)]

$$
\begin{aligned}
\log \left(\frac{\|\|_{\lambda(\xi)}^{2}}{\|\|_{\lambda(\xi)}^{2}}\right)=\frac{1}{|G|} \sum_{g \in G} \log \left(\frac{\|\|_{\lambda(\widetilde{\xi})}^{2}}{\|\|_{\lambda(\tilde{\xi})}^{2}}\right)(g) & =\frac{1}{\left|G / G_{1}\right|} \sum_{h \in G / G_{1}} \log \left(\frac{\|\|_{\lambda\left(\widetilde{\xi}^{G_{1}}\right)}^{\prime 2}}{\|\|_{\lambda\left(\tilde{\xi}^{G_{1}}\right)}^{2}}\right)(h) .
\end{aligned}
$$

Now by [6, Theorem 2.5] and (0.6), we get Theorem 0.1 when $X=\widetilde{X} / G$, and explain that only the maximum proper sub-bundle of $\xi$ plays a role in our formulas (0.1), (0.5).

This paper is organized as follows. In Section 1 we recall some general facts on orbifolds. In Section 2, we construct the Quillen metrics for an orbifold, and prove their anomaly formulas. The last four sections are concerned with the extension of the result of [13] to an orbifold case. In Section 3] we describe the geometric setting of the immersion problem. Also, we extend a result of [13, Theorem 2.1]. In Section 44 by using [11, we construct the Bott-Chern current $T^{\Sigma}\left(\xi, h^{\xi}\right)$. In Section [5, by using the results of [ [5], we establish the corresponding results on the analytic torsion forms associated to a short exact sequence of holomorphic Hermitian orbifold vector bundles. In Section [6] we extend the result of [13] to an orbifold case, i.e. we prove Theorem 0.2 .

In the entire paper we use the superconnection formalism as in [5], 8]. In particular, $\operatorname{Tr}_{s}$ is our notation of the supertrace.

\section{ORBIFOldS AND CHARACTERISTIC CLASSES}

This section is organized as follows. In Section 1.1, we recall the definition of an orbifold [20]. In Section 1.2, we explain some characteristic classes on orbifolds.

1.1. Definition of an orbifold $([20])$. We define a category $\mathcal{M}_{s}$ as follows: The objects of $\mathcal{M}_{s}$ are the class of pairs $(G, M)$ where $M$ is a connected smooth manifold and $G$ is a finite group acting effectively on $M$. Let $(G, M)$ and $\left(G^{\prime}, M^{\prime}\right)$ be two objects, then a morphism $\Phi:(G, M) \rightarrow\left(G^{\prime}, M^{\prime}\right)$ is a family of open embedding $\varphi: M \rightarrow M^{\prime}$ satisfying:

i) For each $\varphi \in \Phi$, there is an injective group homomorphism $\lambda_{\varphi}: G \rightarrow G^{\prime}$ that makes $\varphi$ be $\lambda_{\varphi}$-equivariant.

ii) For $g \in G^{\prime}, \varphi \in \Phi$, we define $g \varphi: M \rightarrow M^{\prime}$ by $(g \varphi)(x)=g \varphi(x)$ for $x \in M$. If $(g \varphi)(M) \cap \varphi(M) \neq \emptyset$, then $g \in \lambda_{\varphi}(G)$.

iii) For $\varphi \in \Phi$, we have $\Phi=\left\{g \varphi, g \in G^{\prime}\right\}$.

Definition 1.1. Let $X$ be a paracompact Hausdorff space and let $\mathcal{U}$ be a covering of $X$ consisting of connected open subsets. We assume $\mathcal{U}$ satisfies the condition:

(1.1) For any $x \in U \cap U^{\prime}, U, U^{\prime} \in \mathcal{U}$, there is $U^{\prime \prime} \in \mathcal{U}$ such that $x \in U^{\prime \prime} \subset U \cap U^{\prime}$.

Then an orbifold structure $\mathcal{V}$ on $X$ is the following:

i) For $U \in \mathcal{U}, \mathcal{V}(U)=\left(\left(G_{U}, \widetilde{U}\right) \stackrel{\tau}{\rightarrow} U\right)$ is a ramified covering $\widetilde{U} \rightarrow U$ giving an identification $U \simeq \widetilde{U} / G_{U}$.

ii) For $U, V \in \mathcal{U}, U \subset V$, there is a morphism $\varphi_{V U}:\left(G_{U}, \widetilde{U}\right) \rightarrow\left(G_{V}, \widetilde{V}\right)$ that covers the inclusion $U \subset V$.

iii) For $U, V, W \in \mathcal{U}, U \subset V \subset W$, we have $\varphi_{W U}=\varphi_{W V} \circ \varphi_{V U}$. 
If $\mathcal{U}^{\prime}$ is a refinement of $\mathcal{U}$ satisfying (1.1), then there is an orbifold structure $\mathcal{V}^{\prime}$ such that $\mathcal{V} \cup \mathcal{V}^{\prime}$ is an orbifold structure. We consider $\mathcal{V}$ and $\mathcal{V}^{\prime}$ to be equivalent. Such an equivalence class is called an orbifold structure over $X$. So we may choose $\mathcal{U}$ arbitrarily fine.

In the above definition, we can replace $\mathcal{M}_{s}$ by a category of manifolds with an additional structure such as orientation, Riemannian metric or complex structure. We understand that the morphisms (and the groups) preserve the specified structure. So we can define oriented, Riemannian or complex orbifolds.

Remark 1.2. Let $P$ be a smooth manifold, and let $H$ be a compact Lie group acting on $P$. We assume that the action of $H$ is locally free. Then the quotient space $P / H$ is an orbifold. Reciprocally, any orbifold $X$ can be presented by this way, with $H=O(n)(n=\operatorname{dim} X)$ [20, p. 76], [22, p. 144].

Let $(X, \mathcal{V})$ be an orbifold. For each $x \in X$, we can choose a small neighborhood $\left(G_{x}, \widetilde{U}_{x}\right) \rightarrow U_{x}$ such that $x \in \widetilde{U}_{x}$ is a fixed point of $G_{x}$. (Such $G_{x}$ is unique up to isomorphisms for each $x \in X$ [30, p. 468]). Let $(1),\left(h_{x}^{1}\right), \cdots,\left(h_{x}^{\rho_{x}}\right)$ be all the conjugacy classes in $G_{x}$. Let $Z_{G_{x}}\left(h_{x}^{j}\right)$ be the centralizer of $h_{x}^{j}$ in $G_{x}$. One also denotes by $\widetilde{U}_{x}^{h_{x}^{j}}$ the fixed points of $h_{x}^{j}$ over $\widetilde{U}_{x}$. Then we have a natural bijection

$$
\left\{\left(y,\left(h_{y}^{j}\right)\right) \mid y \in U_{x}, j=1, \cdots, \rho_{y}\right\} \simeq \coprod_{j=1}^{\rho_{x}} \widetilde{U}_{x}^{h_{x}^{j}} / Z_{G_{x}}\left(h_{x}^{j}\right) .
$$

So we can globally define

$$
\Sigma X=\left\{\left(x,\left(h_{x}^{j}\right)\right) \mid x \in X, G_{x} \neq 1, j=1, \cdots, \rho_{x}\right\} .
$$

Then $\Sigma X$ has a natural orbifold structure defined by

$$
\left\{\left(Z_{G_{x}}\left(h_{x}^{j}\right) / K_{x}^{j}, \widetilde{U}_{x}^{h_{x}^{j}}\right) \rightarrow \widetilde{U}_{x}^{h_{x}^{j}} / Z_{G_{x}}\left(h_{x}^{j}\right)\right\}_{\left(x, U_{x}, j\right)}
$$

Here $K_{x}^{j}$ is the kernel of the representation $Z_{G_{x}}\left(h_{x}^{j}\right) \rightarrow \operatorname{Diffeo}\left(\widetilde{U}_{x}^{h_{x}^{j}}\right)$. The number $m=\left|K_{x}^{j}\right|$ is called the multiplicity of $\Sigma X$ in $X$ at $\left(x, h_{x}^{j}\right)$. Since the multiplicity is locally constant on $\Sigma X$, we may assign the multiplicity $m_{i}$ to each connected component $X_{i}$ of $X \cup \Sigma X$.

Definition 1.3. An orbifold vector bundle $\xi$ over an orbifold $(X, \mathcal{V})$ is defined as follows: $\xi$ is an orbifold and for $U \in \mathcal{U},\left(G_{U}^{\xi}, \widetilde{p}_{U}: \widetilde{\xi}_{U} \rightarrow \widetilde{U}\right)$ is a $G_{U}^{\xi}$-equivariant vector bundle and $\left(G_{U}^{\xi}, \widetilde{\xi}_{U}\right)$ (resp. $\left.\left(G_{U}=G_{U}^{\xi} / K_{U}^{\xi}, \widetilde{U}\right), K_{U}^{\xi}=\operatorname{Ker}\left(G_{U}^{\xi} \rightarrow \operatorname{Diffeo}(\widetilde{U})\right)\right)$ is the orbifold structure of $\xi$ (resp. $X$ ). If $G_{U}^{\xi}$ acts effectively on $\widetilde{U}$ for $U \in \mathcal{U}$, i.e. $K_{U}^{\xi}=\{1\}$, we call $\xi$ a proper orbifold vector bundle.

Remark 1.4. Let $\xi$ be an orbifold vector bundle on $(X, \mathcal{V})$. For $U \in \mathcal{U}$, let $\widetilde{\xi_{U}^{\mathrm{pr}}}$ be the maximum $K_{U}^{\xi}$-invariant sub-bundle of $\widetilde{\xi}_{U}$ on $\widetilde{U}$. Then $\left(G_{U}, \widetilde{\xi_{U}^{\mathrm{pr}}}\right)$ defines a proper orbifold vector bundle on $(X, \mathcal{V})$; we denote it by $\xi^{\mathrm{pr}}$.

Example. The (proper) orbifold tangent bundle $T X$ on an orbifold $X$ is defined by $\left(G_{U}, T \widetilde{U} \rightarrow \widetilde{U}\right)$, for $U \in \mathcal{U}$.

Let $\xi \rightarrow X$ be an orbifold bundle. A section $s: X \rightarrow \xi$ is called $\mathcal{C}^{\infty}\left(\right.$ or $\mathcal{C}^{k}$ ) if for each $U \in \mathcal{U}, s_{\mid U}$ is covered by a $G_{U}^{\xi}$-invariant smooth $\left(\right.$ or $\left.\mathcal{C}^{k}\right)$ section $\widetilde{s}_{U}: \widetilde{U} \rightarrow \widetilde{\xi}_{U}$. 
If $X$ is oriented, we define the integral $\int_{X} \omega$ for a form $\omega$ over $X$ (i.e. a section of $\Lambda\left(T^{*} X\right)$ over $\left.X\right)$ by: if $\operatorname{supp} \omega \subset U \in \mathcal{U}$, then

$$
\int_{X} \omega=\frac{1}{\left|G_{U}\right|} \int_{\widetilde{U}} \widetilde{\omega}_{U}
$$

Definition 1.5. A Kähler form on a complex orbifold $X$ with its complex structure $J$ is a real closed $(1,1)$-form $\omega$ on $X$ such that $\omega(J \cdot, \cdot)$ defines an orbifold metric on $T X$.

In the following, if $G$ does not act effectively on the connected manifold $M$, we will identify the couple $(G, M)$ as an element $(G / K, M)$ in $\mathcal{M}_{s}$, where $K=$ $\operatorname{Ker}(G \rightarrow \operatorname{Diffeo}(M))$.

Definition 1.6. Let $X, Y$ be two orbifolds. The map $i: Y \rightarrow X$ is said to define an orbifold embedding, if $i$ is injective and there exists $\mathcal{U}, \mathcal{U}^{\prime}$ open coverings of $Y$, $X$ such that $\left(G_{U}, \widetilde{U}\right)_{U \in \mathcal{U}},\left(G_{V}, \widetilde{V}\right)_{V \in \mathcal{V}}$ are the orbifold structures of $Y, X$. For $U \in \mathcal{U}$, there is $V \in \mathcal{V}$ such that $U=V \cap Y$ and $\widetilde{i}: \widetilde{U} \rightarrow \widetilde{V}$ is a $G_{V}$-equivariant embedding of $\widetilde{U}$ into $\widetilde{V}$ that covers $i: U \rightarrow V,\left(G_{V}, \widetilde{U}\right)=\left(G_{U}, \widetilde{U}\right)$ in $\mathcal{M}_{s}$. If $U_{1} \subset U_{2}, U_{1}, U_{2} \in \mathcal{U}$, there exist $V_{i} \in \mathcal{V}, U_{i}=V_{i} \cap Y(i=1,2)$, and $V_{1} \subset V_{2}$. We will call $Y$ a sub-orbifold of $X$, and the normal (orbifold) bundle $N$ to $Y$ in $X$ is well defined. Let $K_{U}=\operatorname{Ker}\left\{G_{V} \rightarrow \operatorname{Diffeo}(\widetilde{U})\right\}$. Then $m_{X, Y}=\left|K_{U}\right|$ is locally constant on $Y$; we call $m_{X, Y}$ the relative multiplicity on $Y$.

In particular, if $G$ is a finite group and $\tilde{i}: \widetilde{Y} \rightarrow \widetilde{X}$ is an $G$-equivariant embedding of complex manifolds, then $i: \widetilde{Y} / G \rightarrow \widetilde{X} / G$ is an orbifold embedding.

Definition 1.7. Let $M, B$ be two orbifolds. The map $\pi: M \rightarrow B$ is said to define an orbifold submersion if there exists $\mathcal{U}, \mathcal{U}^{\prime}$ open coverings of $M, B$, such that $\pi(\mathcal{U}) \subset \mathcal{U}^{\prime}$, and $\left(G_{U}, \widetilde{U}\right)_{U \in \mathcal{U}},\left(G_{V}, \widetilde{V}\right)_{V \in \mathcal{V}}$ are the orbifold structures of $M, B$. For $U \in \mathcal{U}$, there is $\widetilde{\pi}: \widetilde{U} \rightarrow \widetilde{V}$ an $G_{U}$-equivariant submersion of $\widetilde{U}$ onto $\widetilde{V}$ that covers $\pi: U \rightarrow V=\pi(U)$, and $\left(G_{U}, \widetilde{V}\right)=\left(G_{V}, \widetilde{V}\right)$ in $\mathcal{M}_{s}$. If $U_{1} \subset U_{2}, U_{1}, U_{2} \in \mathcal{U}$, then $\Phi_{\pi\left(U_{2}\right) \pi\left(U_{1}\right)}$ is induced by $\Phi_{U_{2} U_{1}}$.

Remark 1.8. If $\widetilde{\pi}: \widetilde{M} \rightarrow \widetilde{B}$ is an $G$-equivariant submersion with compact fiber $\widetilde{X}$ (here $G$ is a finite group), then $\pi: \widetilde{M} / G \rightarrow \widetilde{B} / G$ is an orbifold submersion. More generally, if $\pi: M \rightarrow B$ is a proper orbifold submersion from $M$ onto $B$, then for each $b \in B$, there exists a small neighborhood $\left(G_{b}, \widetilde{V}_{b}\right) \rightarrow V_{b}, \widetilde{M}_{b}$ an orbifold, such that $\pi$ is induced by a $G_{b}$-equivariant orbifold submersion $\widetilde{\pi}_{b}: \widetilde{M}_{b} \rightarrow \widetilde{V}_{b}$ with compact fiber $\bar{X}$.

1.2. Characteristic classes. If $A$ is a $(q, q)$ matrix, set

$$
\begin{aligned}
& \operatorname{Td}(A)=\operatorname{det}\left(\frac{A}{1-e^{-A}}\right), \\
& e(A)=\operatorname{det}(A), \quad \operatorname{ch}(A)=\operatorname{Tr}[\exp (A)] .
\end{aligned}
$$

Definition 1.9. Let $P^{X}$ be the vector space of smooth forms on a complex orbifold $X$, which are sums of forms of type $(p, p)$. Let $P^{X, 0}$ be the vector space of the forms $\alpha \in P^{X}$ such that there exist smooth forms $\beta, \gamma$ on $X$ for which $\alpha=\partial \beta+\bar{\partial} \gamma$.

Let $\xi$ be a holomorphic orbifold vector bundle on a complex orbifold $X$. Let $h^{\xi}$ be a Hermitian metric on $\xi$. Then on each local chart, $\left(G_{U}^{\xi}, \widetilde{p}_{U}: \widetilde{\xi}_{U} \rightarrow \widetilde{U}\right)$ in 
Definition 1.3. $h^{\xi}$ lifts to a $G_{U}^{\xi}$-invariant Hermitian metric $h^{\widetilde{\xi}}$ on $\widetilde{\xi}_{U}$. This uniquely defines a holomorphic Hermitian connection $\nabla^{\widetilde{\xi}}$ with curvature $R^{\widetilde{\xi}}$, thus there exists a unique holomorphic Hermitian connection $\nabla^{\xi}$ on $\left(\xi, h^{\xi}\right)$ with curvature $R^{\xi}$. The form $\operatorname{ch}^{\Sigma}\left(\xi, h^{\xi}\right)$ is defined as follows: If $X_{i}$, a connected component of $X \cup \Sigma X$, is represented by $\widetilde{U}^{h}\left(h \in G_{U}\right)$ over $\widetilde{U}$, then $\operatorname{ch}^{\Sigma}\left(\xi, h^{\xi}\right)$ over $\widetilde{U}^{h} / Z_{G_{U}}(h)$ is defined by

$$
\frac{1}{\left|K_{U}^{\xi}\right|} \sum_{g \in \tau^{-1}(h)} \operatorname{Tr}\left[g \exp \left(-\frac{R^{\tilde{\xi}}}{2 \pi i}\right)\right] .
$$

Here $\tau: G_{U}^{\xi} \rightarrow G_{U}=G_{U}^{\xi} / K_{U}^{\xi}$ is the natural projection.

The above definition was justified by the discussions after Theorem 0.2 and (1.6). In fact $h^{\xi}$ induces a Hermitian metric $h^{\xi^{\mathrm{pr}}}$ on the maximum proper orbifold subbundle $\xi^{\mathrm{pr}}$ of $\xi$. Let $\nabla^{\xi^{\mathrm{pr}}}$ be the holomorphic Hermitian connection on $\left(\xi^{\mathrm{pr}}, h^{\xi^{\mathrm{pr}}}\right)$ with curvature $R^{\xi^{\mathrm{pr}}}$. Then on $\widetilde{U}^{h} / Z_{G_{U}}(h)$,

$$
\operatorname{ch}^{\Sigma}\left(\xi, h^{\xi}\right)=\operatorname{Tr}\left[h \exp \left(-\frac{R^{\widetilde{\xi^{\mathrm{pr}}}}}{2 \pi i}\right)\right]=\operatorname{ch}_{h}\left(\widetilde{\xi^{\mathrm{pr}}}, \widetilde{h^{\mathrm{pr}}}\right) .
$$

If $\xi$ is proper, we also define $\operatorname{Td}^{\Sigma}\left(\xi, h^{\xi}\right), \operatorname{Td}^{\Sigma}\left(\xi, h^{\xi}\right)$ on $X \cup \Sigma X$ as follows. Let $1, e^{i \theta_{1}}, \cdots, e^{i \theta_{q}}\left(0<\theta_{j}<2 \pi\right)$ be the locally constant distinct eigenvalues of $h \in G_{U}$ acting on $\widetilde{\xi}$ on $\widetilde{U}^{h}$, and let $\left\{\widetilde{\xi}^{\theta_{i}}\right\}_{i=0}^{q}\left(\theta_{0}=0\right)$ be the corresponding eigenbundles. Then on $\widetilde{U}^{h}, \widetilde{\xi}$ splits holomorphically as an orthogonal sum $\widetilde{\xi}=\bigoplus_{i=0}^{q} \widetilde{\xi}^{\theta_{i}}$, thus $R^{\widetilde{\xi}}=\bigoplus_{i=0}^{q} R^{\tilde{\xi}^{\theta_{i}}}$. We define $\operatorname{Td}^{\Sigma}\left(\xi, h^{\xi}\right), \mathrm{Td}^{\prime \Sigma}\left(\xi, h^{\xi}\right)$ on $\widetilde{U}^{h} / Z_{G_{U}}(h)$ by

$$
\begin{aligned}
& \operatorname{Td}^{\Sigma}\left(\xi, h^{\xi}\right):=\operatorname{Td}_{h}\left(\widetilde{\xi}, h^{\widetilde{\xi}}\right)=\operatorname{Td}\left(\frac{-R^{\tilde{\xi}^{\theta_{0}}}}{2 i \pi}\right) \prod_{j=1}^{q} \frac{\operatorname{Td}}{e}\left(\frac{-R^{\tilde{\xi}^{\theta_{j}}}}{2 i \pi}+i \theta_{j}\right), \\
& \operatorname{Td}^{\prime \Sigma}\left(\xi, h^{\xi}\right):=\operatorname{Td}_{h}^{\prime}\left(\widetilde{\xi}, h^{\widetilde{\xi}}\right)=\frac{\partial}{\partial b}\left[\operatorname{Td}\left(\frac{-R^{\boldsymbol{\xi}_{0}}}{2 i \pi}+b\right) \prod_{j=1}^{q} \frac{\operatorname{Td}}{e}\left(\frac{-R^{\tilde{\xi}_{j}}}{2 i \pi}+i \theta_{j}+b\right)\right]_{b=0} .
\end{aligned}
$$

Then $\operatorname{ch}^{\Sigma}\left(\xi, h^{\xi}\right), \operatorname{Td}^{\Sigma}\left(\xi, h^{\xi}\right), \operatorname{Td}^{\prime \Sigma}\left(\xi, h^{\xi}\right) \in P^{X \cup \Sigma X}$ are closed on $X \cup \Sigma X$ and their cohomology classes do not depend on $h^{\xi}$, and we denote their cohomology classes by $\operatorname{ch}^{\Sigma}(\xi), \operatorname{Td}^{\Sigma}(\xi), \operatorname{Td}^{\Sigma}(\xi) \in H(X \cup \Sigma X, \mathbf{C})$.

Let $h^{\prime \xi}$ be another couple of metrics on $\xi$. Observe that the construction in [8] Remark 1.28, Theorem 1.29] is local and universal, thus we can at first work on each $\widetilde{U}^{h}$, then glue together to get the unique classes $\widetilde{\mathrm{Td}}^{\Sigma}\left(\xi, h^{\xi}, h^{\prime \xi}\right)$ and $\widetilde{\mathrm{ch}}^{\Sigma}\left(\xi, h^{\xi}, h^{\prime \xi}\right)$ in $P^{X \cup \Sigma X} / P^{X \cup \Sigma X, 0}$ such that

$$
\begin{aligned}
& \frac{\bar{\partial} \partial}{2 i \pi} \widetilde{\mathrm{Td}}^{\Sigma}\left(\xi, h^{\xi}, h^{\prime \xi}\right)=\operatorname{Td}^{\Sigma}\left(\xi, h^{\prime \xi}\right)-\operatorname{Td}^{\Sigma}\left(\xi, h^{\xi}\right), \\
& \frac{\bar{\partial} \partial}{2 i \pi} \widetilde{\operatorname{ch}}^{\Sigma}\left(\xi, h^{\xi}, h^{\prime \xi}\right)=\operatorname{ch}^{\Sigma}\left(\xi, h^{\prime \xi}\right)-\operatorname{ch}^{\Sigma}\left(\xi, h^{\xi}\right) .
\end{aligned}
$$

Let $\xi^{i}(0 \leq i \leq m)$ be complex orbifold vector bundles. We assume there is a covering $\mathcal{U}$ satisfying (1.1), such that for $U \in \mathcal{U}$

i) $\left(G_{U}^{\xi}, \bigoplus_{i} \widetilde{\xi}_{U}^{i}\right) \rightarrow \widetilde{U}$ defines an orbifold vector bundle structure $(\xi, \mathcal{V})$;

ii) $G_{U}^{\xi}\left(\widetilde{\xi}_{U}^{i}\right)=\widetilde{\xi}_{U}^{i}$, and $\left(G_{U}^{\xi}, \widetilde{\xi}_{U}^{i}\right)$ induces the orbifold structure of $\xi^{i}$. 
Definition 1.10. We call $v: \xi^{i+1} \rightarrow \xi^{i}$ is a morphism of orbifold vector bundles if there is a $G_{U}^{\xi}$-equivariant morphism $\widetilde{v}: \widetilde{\xi}_{U}^{i+1} \rightarrow \widetilde{\xi}^{i}$ inducing the map $v: \xi^{i+1} \rightarrow \xi^{i}$ on $U$, for each $U \in \mathcal{U}$. We call $\left(\xi^{i}, v\right)$ a complex of orbifold bundles if for each $i$, $v: \xi^{i+1} \rightarrow \xi^{i}$ is a morphism of orbifold vector bundles and $v^{2}=0$. If for each $U \in \mathcal{U},\left(\widetilde{\xi}_{U}^{i}, \widetilde{v}\right)$ is acyclic over $\widetilde{U}$, we call the complex $\left(\xi^{i}, v\right)$ acyclic.

Let $h^{\xi^{i}}$ be Hermitian metrics on $\xi^{i}$. Then $h^{\xi}=\bigoplus h^{\xi^{i}}$ is a Hermitian metric on $\xi$.

If $(\xi, v)$ is an acyclic complex of orbifold bundles, observe that the construction in [8. (1.58), Corollary 1,30] is local and universal. Thus we can first work on each $\widetilde{U}^{h}$ to get $\widetilde{c h}_{h}\left(\xi, h^{\xi}\right)$, which verifies

$$
\frac{\bar{\partial} \partial}{2 i \pi} \widetilde{\operatorname{ch}}_{h}\left(\xi, h^{\xi}\right)=\frac{1}{\left|K_{U}^{\xi}\right|} \sum_{g \in \tau^{-1}(h)}(-1)^{i} \operatorname{Tr}\left[g \exp \left(-\frac{R^{\widetilde{\xi}}}{2 \pi i}\right)\right] .
$$

Then glue together to get a Bott-Chern form $\widetilde{c h}^{\Sigma}\left(\xi, h^{\xi}\right) \in P^{X \cup \Sigma X}$ such that

$$
\frac{\bar{\partial} \partial}{2 i \pi} \widetilde{\operatorname{ch}}^{\Sigma}\left(\xi, h^{\xi}\right)=\sum_{i=0}^{k}(-1)^{i} \operatorname{ch}^{\Sigma}\left(\xi^{i}, h^{\xi^{i}}\right) .
$$

\section{QUillen metrics AND THEIR ANOMALy FORMUlas}

In this section, we construct the Quillen metrics on the inverse of the determinant of the cohomology of a holomorphic orbifold vector bundle, and establish corresponding anomaly formulas. We extend some results of [10] to complex orbifolds.

This section is organized as follows. In Section 2.1, we indicate some properties of elliptic operators over an orbifold. In Section 2.2, by [10], we construct the Quillen metrics. In Section 2.3, we prove our anomaly formulas.

In this section, we use the notation of Section 1.1.

2.1. Elliptic operator over an orbifold. Let $(X, \mathcal{V})$ be a compact oriented Riemannian orbifold of real dimension $n$. For $x, y \in X$, put

$$
\begin{gathered}
d^{X}(x, y)=\operatorname{Inf}_{\gamma}\left\{\sum_{i} \int_{t_{i-1}}^{t_{i}}\left|\frac{\partial}{\partial t} \widetilde{\gamma}_{i}(t)\right| d t \mid \gamma:[0,1] \rightarrow X, \gamma(0)=x, \gamma(1)=y,\right. \text { such that } \\
\quad \text { there exist } t_{0}=0<t_{1}<\cdots<t_{k}=1, U_{i} \in \mathcal{U}, \gamma\left(\left[t_{i-1}, t_{i}\right]\right) \subset U_{i}, \\
\left.\quad \text { and a } \mathcal{C}^{\infty} \text { map } \widetilde{\gamma}_{i}:\left[t_{i-1}, t_{i}\right] \rightarrow \widetilde{U}_{i} \text { that covers } \gamma_{\mid\left[t_{i-1}, t_{i}\right]}\right\} .
\end{gathered}
$$

Then $\left(X, d^{X}\right)$ is a metric space.

Let $\left(\xi, h^{\xi}\right),\left(\eta, h^{\eta}\right)$ be (complex) Hermitian orbifold vector bundles over $X$ and for $U \in \mathcal{U}$, let $\left(G_{U}, \widetilde{U}\right) \stackrel{\tau}{\rightarrow} U,\left(G_{U}^{\xi}, \widetilde{p}_{U}: \widetilde{\xi}_{U} \rightarrow \widetilde{U}\right),\left(G_{U}^{\eta}, \widetilde{p}_{U}: \widetilde{\eta}_{U} \rightarrow \widetilde{U}\right)$ be defined in Definition 1.3. We assume that for $U \in \mathcal{U}, G_{U}^{\xi}=G_{U}^{\eta}$. Let

$$
K_{U}^{\xi}=\operatorname{Ker}\left(G_{U}^{\xi} \rightarrow \operatorname{Diffeo}(\widetilde{U})\right) .
$$

We denote by $\widetilde{d}(\cdot, \cdot)$ the distance on $\widetilde{U}$ with respect to $g^{T X}$.

Let $d v_{X}$ be the Riemannian volume element on $X$ with respect to the metric $g^{T X}$. Let $\mathrm{pr}_{1}$ and $\mathrm{pr}_{2}$ be the projections from $X \times X$ onto the first and second factor $X$, respectively. We denote by $\mathcal{C}^{k}(X, \xi)(k \in \mathbf{N})$ the $\mathcal{C}^{k}$ sections of $\xi$ on $X$. A $\mathcal{C}^{k}$ section $K(x, y)$ of $\operatorname{pr}_{1}^{*} \eta \otimes \operatorname{pr}_{2}^{*} \xi^{*}$ on $X \times X$ gives an operator $T: \mathcal{C}^{0}(X, \xi) \rightarrow \mathcal{C}^{k}(X, \eta)$ 
defined by

$$
T \sigma(x)=\int_{X} K(x, y) \sigma(y) d v_{X}(y)
$$

We call $K$ the kernel of the operator $T$ and we call $T$ a smoothing operator if $k=+\infty$.

Let $D: \mathcal{C}^{\infty}(X, \xi) \rightarrow \mathcal{C}^{\infty}(X, \eta)$ be a linear map. We call $D$ a differential operator of order $m$ if for each $U \in \mathcal{U}, D$ is presented by a $G_{U}^{\xi}$-invariant differential operator $\widetilde{D}_{U}: \mathcal{C}^{\infty}(\widetilde{U}, \widetilde{\xi}) \rightarrow \mathcal{C}^{\infty}(\widetilde{U}, \widetilde{\eta})$ of order $m$. We call $D$ a pseudo-differential operator of order $m$ if for each $U \in \mathcal{U}$, there is a $G_{U}^{\xi}$-invariant pseudo-differential operator $\widetilde{D}_{U}: \mathcal{C}^{\infty}(\widetilde{U}, \widetilde{\xi}) \rightarrow \mathcal{C}^{\infty}(\widetilde{U}, \widetilde{\eta})$ of order $m$ such that: For any smooth partition of unity $\left\{\varphi_{U}\right\}$ subordinate to $\mathcal{U}$ and a family $\left\{\psi_{U}\right\}$ of smooth functions satisfying $\varphi_{U} \psi_{U}=\varphi_{U}$ and $\operatorname{supp}\left\{\psi_{U}\right\} \in U$, the difference $D-\Sigma_{U} \varphi_{U} \widetilde{D}_{U} \psi_{U}$ is a smoothing operator. If each $\widetilde{D}_{U}$ is elliptic, we call $D$ elliptic. For an elliptic pseudo-differential operator, we can construct a parametrix [20, p. 82]. If $D$ is also positive and $\xi=\eta$, we can construct $P_{t}(x, y) \in \mathcal{C}^{\infty}\left(X \times X, \operatorname{pr}_{1}^{*} \xi \otimes \operatorname{pr}_{2}^{*} \xi^{*}\right)(t>0)$ as the kernel of the heat operator $e^{-t D}: \mathcal{C}^{\infty}(X, \xi) \rightarrow \mathcal{C}^{\infty}(X, \xi)$ with respect to $d v_{X}$ as in [25, §3.6].

Before we state the asymptotic expansion of the heat kernel as $t \rightarrow 0$, we indicate if $D$ is a pseudo-differential operator of order $m(m<-n-k, k \in \mathbf{N})$. Then the operator $D$ has a $\mathcal{C}^{k}$-kernel. In fact, if $\widetilde{D}_{U}$ has a $\mathcal{C}^{k}$-kernel $\widetilde{Q}_{U}\left(\widetilde{x}_{1}, \widetilde{x}_{2}\right)$ over $\widetilde{U} \times \widetilde{U}$, then for $x_{1}, x_{2} \in U$,

$$
Q_{U}\left(x_{1}, x_{2}\right)=\frac{1}{\left|K_{U}^{\xi}\right|} \sum_{g \in G_{U}^{\xi}}(g, 1) \widetilde{Q}_{U}\left(g^{-1} \widetilde{x}_{1}, \widetilde{x}_{2}\right)
$$

is the kernel of the operator $D_{U}: \mathcal{C}^{\infty}\left(U,\left.\xi\right|_{U}\right) \rightarrow \mathcal{C}^{\infty}\left(U,\left.\eta\right|_{U}\right)$, with $\tau\left(\widetilde{x}_{i}\right)=x_{i}(i=$ $1,2)$. Indeed, for $s \in C^{\infty}\left(U,\left.\xi\right|_{U}\right)$ with compact support, by definition,

$$
\begin{aligned}
\left(Q_{U} s\right)\left(x_{1}\right) & =\left(\widetilde{Q}_{U} \widetilde{s}\right)\left(\widetilde{x}_{1}\right)=\int_{\widetilde{U}} \widetilde{Q}_{U}\left(\widetilde{x}_{1}, \widetilde{x}_{2}\right) \widetilde{s}\left(\widetilde{x}_{2}\right) d v_{\widetilde{U}}\left(\widetilde{x}_{2}\right) \\
& =\frac{1}{\left|G_{U}^{\xi}\right|} \sum_{g \in G_{U}^{\xi}} \int_{\widetilde{U}}(g, 1) \widetilde{Q}_{U}\left(g^{-1} \widetilde{x}_{1}, \widetilde{x}_{2}\right) \widetilde{s}\left(\widetilde{x}_{2}\right) d v_{\widetilde{U}}\left(\widetilde{x}_{2}\right) .
\end{aligned}
$$

Proposition 2.1. Let $H$ be a second order differential operator on $\xi$ such that $\sigma_{2}(H)(x, u)=|u|^{2}\left(u \in T^{*} X\right)$. Let $P_{t}(x, y)$ be the kernel of the heat operator $e^{-t H}$ with respect to $d v_{X}$. Then for each $U \in \mathcal{U}$, there exists a smooth section $\Phi_{i} \in \mathcal{C}^{\infty}\left(\widetilde{U} \times \widetilde{U}, \operatorname{pr}_{1}^{*} \widetilde{\xi} \otimes \operatorname{pr}_{2}^{*} \widetilde{\xi}^{*}\right)$ such that for every $k>n, x, y \in U$, as $t \rightarrow 0$

$$
P_{t}(x, y)=(4 \pi t)^{-n / 2} \frac{1}{\left|K_{U}^{\xi}\right|} \sum_{g \in G_{U}^{\xi}} \sum_{i=0}^{k}(g, 1) e^{\frac{-\tilde{d}\left(g^{-1} \tilde{x}, \tilde{y}\right)^{2}}{4 t}} t^{i} \Phi_{i}\left(g^{-1} \widetilde{x}, \widetilde{y}\right)+O\left(t^{k-n / 2}\right) .
$$

On $\{(x, y) \in X \times X, d(x, y)>c>0\}$, as $t \rightarrow 0$, we have $P_{t}(x, y)=O\left(e^{-c^{2} / 4 t}\right)$.

Proof. By proceeding as in [2, §2.4, 2.5$]$ or proceeding as in [31, II §7.13], and using (2.2), we have Proposition 2.1.

2.2. Quillen metrics. Let $X$ be a compact complex orbifold of complex dimension

$l$. Let $\xi$ be a holomorphic orbifold vector bundle on $X$.

Let $\mathcal{O}_{X}$ be the sheaf over $X$ of local $G_{U}$-invariant holomorphic functions over $\widetilde{U}$, for $U \in \mathcal{U}$. Then by [15], $\left(X, \mathcal{O}_{X}\right)$ is an analytic space. The local $G_{U}^{\xi}$-invariant 
holomorphic sections of $\widetilde{\xi} \rightarrow \widetilde{U}$ also define a coherent analytic sheaf $\mathcal{O}_{X}(\xi)$ over $X$ and as in (1.6),

$$
\mathcal{O}_{X}(\xi)=\mathcal{O}_{X}\left(\xi^{\mathrm{pr}}\right)
$$

Let $\mathcal{D}^{p}(\xi)$ be the sheaf of $\mathcal{C}^{\infty}$ sections of $\Lambda^{p}\left(T^{*(0,1)} X\right) \otimes \xi$ over $X$. Then we have the Dolbeault operator $\bar{\partial}^{X}: \mathcal{D}^{p}(\xi) \rightarrow \mathcal{D}^{p+1}(\xi)$ and an exact sequence of $\mathcal{O}_{X}$-sheaves

$$
0 \rightarrow \mathcal{O}_{X}(\xi) \rightarrow \mathcal{D}^{1}(\xi) \stackrel{\bar{\partial}^{X}}{\rightarrow} \cdots \stackrel{\bar{\partial}^{X}}{\rightarrow} \mathcal{D}^{l}(\xi) \rightarrow 0 .
$$

Put

$$
\Omega^{p}(X, \xi)=\Gamma\left(X, \mathcal{D}^{p}(\xi)\right), \quad \Omega^{\bullet}(X, \xi)=\bigoplus_{p} \Omega^{p}(X, \xi) .
$$

Then we have $\left(\Omega^{\bullet}(X, \xi), \bar{\partial}^{X}\right)$, the Dolbeault complex of $\mathcal{C}^{\infty}$ sections of $\Lambda\left(T^{*(0,1)} X\right)$ $\otimes \xi$ over $X$ :

$$
0 \rightarrow \Omega^{0}(X, \xi) \stackrel{\bar{\partial}^{X}}{\rightarrow} \cdots \stackrel{\bar{\partial}^{X}}{\rightarrow} \Omega^{l}(X, \xi) \rightarrow 0 .
$$

The sheaves $\mathcal{D}^{p}(\xi)$ are fine [21], so their higher cohomology groups vanish. Thus

$$
H\left(\Omega^{\bullet}(X, \xi), \bar{\partial}^{X}\right) \simeq H\left(X, \mathcal{O}_{X}(\xi)\right) .
$$

In what follows, we also denote $H\left(X, \mathcal{O}_{X}(\xi)\right)$ by $H(X, \xi)$.

Let $g^{T X}, h^{\xi}$ be Hermitian metrics on $T X, \xi$. Let $d v_{X}$ be the Riemannian volume form on $X$ associated to $g^{T X}$. Let \langle\rangle$_{\Lambda\left(T^{*(0,1)} X\right) \otimes \xi}$ be the Hermitian product induced by $g^{T X}, h^{\xi}$ on $\Lambda\left(T^{*(0,1)} X\right) \otimes \xi$. The Hermitian product $\left\langle>\right.$ on $\Omega^{\bullet}(X, \xi)$ is defined by: If $s, s^{\prime} \in \Omega^{\bullet}(X, \xi)$, set

$$
\left\langle s, s^{\prime}\right\rangle=\left(\frac{1}{2 \pi}\right)^{\operatorname{dim} X} \int_{X}\left\langle s, s^{\prime}\right\rangle_{\Lambda\left(T^{*(0,1)} X\right) \otimes \xi}(x) d v_{X}(x) .
$$

Let $\bar{\partial}^{X *}$ be the formal adjoint of $\bar{\partial}^{X}$ with respect to the Hermitian product (2.8) associated to $g^{T X}, h^{\xi}$. Set

$$
D^{X}=\bar{\partial}^{X}+\bar{\partial}^{X *}, \quad K^{\bullet}(X, \xi)=\operatorname{Ker} D^{X} .
$$

Then $D^{X, 2}=\bar{\partial}^{X} \bar{\partial}^{X *}+\bar{\partial}^{X *} \bar{\partial}^{X}$ preserves $\mathbf{Z}$-grading of $\Omega(X, \xi)$.

Proposition 2.2 (The Hodge Decomposition Theorem). There is an $L_{2}$-orthogonal direct sum decomposition of the $\xi$-value $(0, p)$-forms

$$
\Omega^{p}(X, \xi)=K^{\bullet}(X, \xi) \oplus \operatorname{Im} \bar{\partial}^{X} \oplus \operatorname{Im} \bar{\partial}^{X *} .
$$

Proof. On each local chart $U \in \mathcal{U}$, the lifting of $\widetilde{D_{U}^{X}}$ on $\widetilde{U}$ is elliptic and $G_{U^{-}}^{\xi}$ invariant, and $\sigma\left({\widetilde{D_{U}^{X}}}^{2}\right)(u)=|u|^{2}$. Thus $D^{X}$ is a self-adjoint elliptic operator. By [20, p. 82], we can construct a parametrix for $D^{X}$. Now, we can proceed as in [25. Theorems 3.5.5-3.5.7], and we know that there is an $L_{2}$-orthogonal direct decomposition

$$
\Omega(X, \xi)=\operatorname{Ker} D^{X, 2} \oplus \operatorname{Im} D^{X, 2} .
$$

In fact $\operatorname{Ker} D^{X}=\operatorname{Ker} D^{X, 2}$, so $\operatorname{Ker} D^{X, 2}, \operatorname{Im} \bar{\partial}^{X}, \operatorname{Im} \bar{\partial}^{X *}$ are orthogonal. Clearly, $\operatorname{Im} D^{X, 2} \subset \operatorname{Im} \bar{\partial}^{X}+\operatorname{Im} \bar{\partial}^{X *}$. Using (2.11), we have Proposition [2.2. 
From (2.7), 2.10), and (2.11), there is a canonical identification

$$
K^{\bullet}(X, \xi) \simeq H^{\bullet}(X, \xi) .
$$

Let $h^{H(X, \xi)}$ be the corresponding metric on $H(X, \xi)$ induced by the restriction of the $L_{2}$-metric (2.8) to $K(X, \xi)$ via the canonical isomorphism (2.12).

Let $\lambda(\xi)$ be the inverse of the determinant of the cohomology of $\xi$ on $X$,

$$
\operatorname{det} H(X, \xi)=\bigotimes_{i=0}^{\operatorname{dim} X}\left(\operatorname{det} H^{i}(X, \xi)\right)^{(-1)^{i}}, \quad \lambda(\xi)=(\operatorname{det} H(X, \xi))^{-1} .
$$

Let ||$_{\lambda(\xi)}$ be the metric on $\lambda(\xi)$ induced by $h^{H(X, \xi)}$. The metric ||$_{\lambda(\xi)}$ will be called the $L_{2}$-metric on $\lambda(\xi)$.

Let $P$ be the orthogonal projection operator from $\Omega^{\bullet}(X, \xi)$ on $K(X, \xi)$ with respect to the Hermitian product (2.8). Set $P^{\perp}=1-P$. Let $\mathrm{N}$ be the number operator defining the $\mathbf{Z}$-grading of $\Omega^{\bullet}(X, \xi)$, i.e. $\mathrm{N}$ acts by multiplication by $k$ on $\Omega^{k}(X, \xi)$. For $s \in \mathbf{C}, \operatorname{Re}(s)>\operatorname{dim} X$, set

$$
\theta^{\xi}(s)=-\operatorname{Tr}_{s}\left[\mathrm{~N}\left(D^{X, 2}\right)^{-s} P^{\perp}\right]=\frac{-1}{\Gamma(s)} \int_{0}^{+\infty} t^{s-1} \operatorname{Tr}_{s}\left[\mathrm{~N} \exp \left(-t D^{X, 2}\right) P^{\perp}\right] d t .
$$

From (2.3), (2.14), $\theta^{\xi}(s)$ extends to a meromorphic function of $s \in \mathbf{C}$ which is holomorphic at $s=0$.

Following [28], [10], we now define the Quillen metric on the line $\lambda(\xi)$.

Definition 2.3. Let \|\|$_{\lambda(\xi)}$ be the Quillen metric on the line $\lambda(\xi)$,

$$
\|\quad\|_{\lambda(\xi)}=|\quad|_{\lambda(\xi)} \exp \left(-\frac{1}{2} \frac{\partial \theta^{\xi}}{\partial s}(0)\right)
$$

2.3. Anomaly formulas for Quillen metrics. Let $g^{\prime T X}, h^{\prime \xi}$ be another couple of metrics on $T X, \xi$. We denote with a ' the objects attached to $g^{\prime T X}, h^{\prime \xi}$. Let $\widetilde{\operatorname{Td}}^{\Sigma}\left(T X, g^{T X}, g^{\prime T X}\right)$ and $\widetilde{c h}^{\Sigma}\left(\xi, h^{\xi}, h^{\prime \xi}\right)$ in $P^{X \cup \Sigma X} / P^{X \cup \Sigma X, 0}$ be defined as in (1.8). Let $m_{i}$ be the multiplicity of each connected component $X_{i}$ of $X \cup \Sigma X$.

Proof of Theorem 0.1. From (1.6), (2.4), (2.6), we only need to prove Theorem 0.1 for a proper orbifold vector bundle. Assume now that $\xi$ is a proper orbifold vector bundle. Let $c \in[0,1] \rightarrow\left(g_{c}^{T X}, h_{c}^{\xi}\right)$ be a smooth family of Hermitian metrics on $T X, \xi$ such that for any $c, g_{c}^{T X}$ is Kähler and also $\left(g_{0}^{T X}, h_{0}^{\xi}\right)=\left(g^{T X}, h^{\xi}\right)$, $\left(g_{1}^{T X}, h_{1}^{\xi}\right)=\left(g^{\prime T X}, h^{\prime \xi}\right)$. Let \|\|$_{\lambda(\xi), c}$ be the corresponding Quillen metric on $\lambda(\xi)$.

Let $\bar{\partial}_{c}^{X *}$ be the adjoint of $\bar{\partial}^{X}$ with respect to $\left(g_{c}^{T X}, h_{c}^{\xi}\right)$. Set $D_{c}^{X}=\bar{\partial}^{X}+\bar{\partial}_{c}^{X *}$. Let $*_{c}$ be the Hodge star operator attached to $g_{c}^{T X}$. Set

$$
Q_{c}=-*_{c}^{-1} \frac{\partial *_{c}}{\partial c}-\left(h_{c}^{\xi}\right)^{-1} \frac{\partial h_{c}^{\xi}}{\partial c} .
$$

Using (2.3), as $t \rightarrow 0$, we have an asymptotic expansion

$$
\operatorname{Tr}_{s}\left[Q_{c} \exp \left(-t D_{c}^{X, 2}\right)\right]=\sum_{j=-l}^{0} M_{j, c} t^{j}+O(t) .
$$


By proceeding formally as in [10, Theorem 1.18], we get

$$
\frac{\partial}{\partial c} \log \left(\frac{\|\|_{\lambda(\xi), c}^{2}}{\|\|_{\lambda(\xi)}^{2}}\right)=M_{0, c} .
$$

Assume first that $h_{c}^{\xi}=h^{\xi}=h^{\prime \xi}$. Let $d a, d \bar{a}$ be two odd Grassmann variables. In particular, $d a, d \bar{a}$ anticommute with the operator $D^{X}$. Set

$$
L_{t}=-t D^{X, 2}-\sqrt{\frac{t}{2}} d a D^{X}-\sqrt{\frac{t}{2}} d \bar{a}\left[D^{X}, Q\right]+d a d \bar{a} Q .
$$

If $\alpha \in \mathbf{C}(d a, d \bar{a})$, let $[\alpha]^{d a} d \bar{a} \in \mathbf{C}$ be the coefficient of $d a d \bar{a}$ in the expansion of $\alpha$. Proceeding formally as in [10, Theorem 1.20], when $t \rightarrow 0$, we get

$$
\operatorname{Tr}_{s}\left[\exp \left(L_{t}\right)\right]^{d a d \bar{a}}=M_{0}+O(t) .
$$

Let $P_{t}\left(x, x^{\prime}\right)\left(x, x^{\prime} \in X\right)$ be the smooth kernel associated to $\exp \left(L_{t}\right)$ with respect to $d v_{X} /(2 \pi)^{\operatorname{dim} X}$. Then

$$
\operatorname{Tr}_{s}\left[\exp \left(L_{t}\right)\right]=\int_{X} \operatorname{Tr}_{s}\left[P_{t}(x, x)\right] d v_{X} /(2 \pi)^{\operatorname{dim} X}
$$

By Section 1.1, for each $x \in X$, we choose a small neighborhood $\left(G_{x}, \widetilde{U}_{x}\right) \rightarrow U_{x}$, such that $\widetilde{x}=\tau^{-1}(x)$ is a fixed point of $G_{x}$. For $\alpha>0$, we denote $B(\widetilde{x}, \alpha) \subset \widetilde{U}_{x}$ as the ball with the center $\widetilde{x}$ and radius $\alpha$. For $\alpha$ small enough, there exist $x_{i} \in X$ $(i \in I=\{1, \cdots, k\})$ such that $B\left(\widetilde{x}_{i}, \alpha\right) \subset \widetilde{U}_{x_{i}}$ and $\left(G_{x_{i}}, B\left(\widetilde{x}_{i}, \alpha / 2\right)\right)$ is a covering of $X$. Let $\rho_{x_{i}}$ be a partition of unity subordinate to this covering.

Now, near $x_{i} \in X$, we replace $X$ by $\mathbf{C}^{l} / G_{x_{i}}$, with $0 \in T_{x_{i}} \widetilde{X}$ representing $\widetilde{x_{i}}$, and the extended fibrations over $\mathbf{C}^{l}$ coincide with given fibration on $B(0, \alpha / 2)$. Let $d v_{T_{x_{i}} \widetilde{X}}(y)$ be the Riemannian volume form on $\left(T_{\mathbf{R}, x_{i}} \widetilde{X}, h^{T_{\mathbf{R}, x_{i}} \widetilde{X}}\right) \simeq \mathbf{R}^{2 l}$. For $y \in \mathbf{C}^{l},|y|<\alpha / 2$, set

$$
d v_{\widetilde{X}}(y)=k(y) d v_{T_{x_{i}} \widetilde{X}}(y)
$$

Let $\widetilde{P}_{u}(x, y)\left(x, y \in T_{\mathbf{R}} \widetilde{X}=\mathbf{C}^{n}\right)$ be the kernel of $\exp \left(L_{t}\right)$ with respect to $d v_{T_{x_{i}} \widetilde{X}}(y) /$ $(2 \pi)^{\operatorname{dim} X}$. By (2.2), and using finite propagation speed as in $\left.\left.7, \S 11 \mathrm{~b}\right)\right]$ (cf. (6.34)), we get

$$
\begin{aligned}
& \lim _{u \rightarrow 0} \int_{X} \rho_{x_{i}} \operatorname{Tr}_{s}\left[P_{u}(y, y)\right] d v_{X}(y) /(2 \pi)^{\operatorname{dim} X} \\
& \quad=\lim _{u \rightarrow 0} \int_{\widetilde{U}_{x_{i}}} \rho_{x_{i}} \frac{1}{\left|G_{x_{i}}\right|} \sum_{g \in G_{x_{i}}} \operatorname{Tr}_{s}\left[g \widetilde{P}_{u}\left(g^{-1} y, y\right)\right] k(y) d v_{T_{x_{i}} \widetilde{X}}(y) /(2 \pi)^{\operatorname{dim} X} .
\end{aligned}
$$

Let $1, e^{i \theta_{1}}, \cdots, e^{i \theta_{q}}\left(0<\theta_{j}<2 \pi\right)$ be the locally constant distinct eigenvalues of $g$ acting on $T \widetilde{X}$ over $T_{x_{i}} \widetilde{X}^{g}$. Let $\widetilde{N}^{\theta_{j}}$ be the corresponding eigenbundles. Let $R^{T \widetilde{X}^{g}}, R^{\widetilde{N}^{\theta_{j}}}$ be their curvatures as in Section [1.2, By [27, (2.56)] or [6, (2.63)], we 
have

$$
\begin{aligned}
& \lim _{u \rightarrow 0} \int_{X} \rho_{x_{i}} \operatorname{Tr}_{s}\left[P_{u}(y, y)\right]^{d a d \bar{a}} d v_{X}(y) /(2 \pi)^{\operatorname{dim} X} \\
& =\frac{1}{\left|G_{x_{i}}\right|} \sum_{g \in G_{x_{i}}} \int_{\widetilde{U}_{x_{i}}^{g}} \rho_{x_{i}} \frac{\partial}{\partial b}\left[\operatorname{Td}\left(\frac{-R^{T \widetilde{X}^{g}}}{2 i \pi}-b\left(h^{T \widetilde{X}}\right)^{-1} \frac{\partial h^{T \widetilde{X}}}{\partial c}\right)\right. \\
& \left.\quad \times \prod_{j=1}^{q} \frac{\operatorname{Td}}{e}\left(\frac{-R^{\widetilde{N}^{\theta_{j}}}}{2 i \pi}-b\left(h^{\widetilde{N}^{\theta_{j}}}\right)^{-1} \frac{\partial h^{\widehat{N}_{j}}}{\partial c}+i \theta_{j}\right)\right]_{b=0} \operatorname{ch}_{g}\left(\xi, h^{\xi}\right) .
\end{aligned}
$$

By using Section 1.2, we finish the proof of Theorem 0.1 .

Assume now that $g_{c}^{T X}=g^{T X}=g^{\prime T X}$. Then since $g^{T X}$ is Kähler, we may use the local index technique as above, and find that we have the analogue of [6, (2.41), $(2.42)$ ], so we get (0.1).

Let $\omega^{X}$ be the Kähler form on $X$ associated to $g^{T X}$ as in Definition 1.5. Set

$$
\begin{aligned}
& C_{-1}=\sum_{i} \frac{1}{m_{i}} \int_{X_{i}} \frac{\omega^{X}}{2 \pi} \operatorname{Td}^{\Sigma}\left(T X, g^{T X}\right) \operatorname{ch}^{\Sigma}\left(\xi, h^{\xi}\right) \\
& C_{0}=\sum_{i} \frac{1}{m_{i}} \int_{X_{i}}\left(-\operatorname{Td}^{\Sigma}\left(T X, g^{T X}\right)+\operatorname{dim} X \operatorname{Td}^{\Sigma}\left(T X, g^{T X}\right)\right) \operatorname{ch}^{\Sigma}\left(\xi, h^{\xi}\right) .
\end{aligned}
$$

Theorem 2.4. As $u \rightarrow 0$,

$$
\operatorname{Tr}_{s}\left[N \exp \left(-u D^{X, 2}\right)\right]=\frac{1}{u} C_{-1}+C_{0}+O(u) .
$$

Proof. For $c>0$, set $g_{c}^{T X}=g^{T X} / c, h_{c}^{\xi}=h^{\xi}$ in the proof of Theorem 0.1. Let $*_{c}$ be the star operators associated to the metrics $g_{c}^{T X}$. Clearly, when acting on $\Lambda\left(T^{*(0,1)} X\right) \otimes \xi, *_{c}=c^{-\operatorname{dim} X} *_{1} c^{N}$, and so

$$
\left(*_{c}\right)^{-1} \frac{\partial *_{c}}{\partial c}=(N-\operatorname{dim} X) / c
$$

Now, by [6. (2.44)], (2.23) and (2.26) for $c=1$, we get (2.25).

\section{COMPleX ORBIFOld IMMERSiON, ORBIFOLD RESOlUtion AND QUILLEN METRICS}

In this section, we introduce our basic setting. This section extends [13, §1] to the orbifold case.

This section is organized as follows. In Section 3.1, we introduce our basic geometric setting. In Section 3.2, we construct a Quillen metric on an intermediate object $\widetilde{\lambda}(\xi)$. In Section 3.3 we state an extension of [13, Theorem 2.1]. In Section 3.4, we make various assumptions on the metrics on $T X, \xi, T Y, \eta$.

3.1. Complex orbifold immersions and resolutions. Let $(X, \mathcal{V})$ be a compact connected complex orbifold of complex dimension $l$. Let $(Y, \mathcal{U})=\bigcup_{j=1}^{d} Y^{j}$ be a finite union of compact connected sub-orbifolds of $X$ such that for $1 \leq j<j^{\prime} \leq$ $d, Y^{j} \cap Y^{j^{\prime}}=\emptyset$. Let $i$ be the embedding $Y \rightarrow X$. For $1 \leq j \leq d$, let $l_{j}^{\prime}$ be the complex dimension of $Y^{j}$. 
Let $\eta$ be a holomorphic orbifold vector bundle on $Y$. For $1 \leq j \leq d$, let $\eta^{j}$ be the restriction of $\eta$ to $Y^{j}$. Let

$$
(\xi, v): 0 \rightarrow \xi_{m} \rightarrow \cdots \rightarrow \xi_{0} \rightarrow 0
$$

be a holomorphic chain complex of orbifold vector bundles on $X$ (cf. Section 1.2). In what follows, we identify $\xi$ with $\bigoplus_{k=0}^{m} \xi_{k}$. Let $r$ be a holomorphic orbifold restriction map $\left.\xi_{0}\right|_{Y} \rightarrow \eta$.

Definition 3.1. The complex $(\xi, v)$ is said to be an orbifold resolution of the sheaf $i_{*} \mathcal{O}_{Y}(\eta)$, if for each $U \in \mathcal{U}$, there exists $V \in \mathcal{V}$ such that $U=V \cap Y$ and over $\widetilde{V}$, we have a $G_{V}^{\xi}$-equivariant exact sequence of sheaves

$$
0 \rightarrow \mathcal{O}_{\widetilde{V}}\left(\widetilde{\xi}_{m}\right) \stackrel{v}{\rightarrow} \cdots \stackrel{v}{\rightarrow} \mathcal{O}_{\widetilde{V}}\left(\widetilde{\xi}_{0}\right) \stackrel{r}{\rightarrow} i_{*} \mathcal{O}_{\widetilde{U}}(\widetilde{\eta}) \rightarrow 0 .
$$

Let $(\xi, v)$ be an orbifold resolution of the sheaf $i_{*} \mathcal{O}_{Y}(\eta)$. Let $H(X, \xi)$ be the hypercohomology of $\left(\mathcal{O}_{X}(\xi), v\right)$. Set (cf. (2.13) $)$

$$
\begin{aligned}
& \lambda\left(\xi_{i}\right)=\left(\operatorname{det} H\left(X, \xi_{i}\right)\right)^{-1}, \quad \lambda(\xi)=\otimes_{i=0}^{m}\left(\lambda\left(\xi_{i}\right)\right)^{(-1)^{i}}, \\
& \tilde{\lambda}(\xi)=(\operatorname{det} H(X, \xi))^{-1}, \quad \lambda(\eta)=(\operatorname{det} H(Y, \eta))^{-1} .
\end{aligned}
$$

Now, we recall the construction of the canonical nonzero section of the line $\lambda^{-1}(\eta) \otimes \lambda(\xi)$ [23]. Let $F(\cdot)=\Gamma(X, \cdot)$ be the global section functor over $\mathcal{O}_{X^{-}}$ sheaves on $X$. From [17, pp. 146-147], we can construct two derived spectral functors of $F$ on the category of complexes of $\mathcal{O}_{X}$-sheaves on $X$.

Let $K=\left(K_{i}, v\right)$ be a bounded complex of $\mathcal{O}_{X}$-sheaves on $X$. Let $H^{i}(K)$ be the $i^{t h}$ cohomology group of $(K, v)$. Let $H(X, K)$ be the hypercohomology of $K$. The two spectral sequence functors ' $E_{r}(K)$ and ${ }^{\prime \prime} E_{r}(K)(r \geq 2)$ associated to $K$ abuts to $H(X, K)$ (associated to filtration of $H(X, K)$ ), and

$$
{ }^{\prime} E_{2}^{p, q}(K)=H^{p}\left(H^{q}(X, K .), v\right), \quad{ }^{\prime \prime} E_{2}^{p, q}(K)=H^{p}\left(X, H^{q}(K)\right) .
$$

Definition $3.2([16$, Chap. XVII]). An (injective) resolution of a complex $K=$ $\left(K_{i}, v\right)$ is a double complex $L=\left(L^{p, q}\right)$ defined in degrees $\geq 0$, together with a morphism $\varepsilon: K \rightarrow L^{\bullet, 0}$, such that $L^{p, q}$ is an (injective) $\mathcal{O}_{X}$-sheave for each $p, q \geq 0$, and for $p$ fixed, if we take the cycles $Z^{p, q}$ (resp. boundaries $B^{p, q}$, resp. cohomologies $H^{p, q}$ ) of $L^{p, *}$ with respect to the first differential operator, we find (injective) resolution of the cycle (resp. boundary, resp. cohomology) in $K^{p}$.

Let $L=\left(L^{p, q}\right)$ be an injective resolution of $K$. Then the first (resp. second) spectral sequence of the double complex $F(L)$ calculates the spectral sequence ${ }^{\prime} E_{r}(K)$ (resp. " $\left.E_{r}(K)\right)(r \geq 2)$. By [17, p. 147], to calculate the spectral sequence ${ }^{\prime} E_{r}(K)$ (resp. " $\left.E_{r}(K)\right)(r \geq 2)$, we can take $L^{p, q}$ such that $Z^{p, q}, B^{p, q}, H^{p, q}$ are $F_{*}$-acyclic.

Let $K=\left(K_{i}, v\right)_{0 \leq i \leq m}$ be a complex of coherent $\mathcal{O}_{X}$-sheaves on $X$. Set

$$
\begin{aligned}
& \lambda\left(K_{i}\right)=\left(\operatorname{det} H\left(X, K_{i}\right)\right)^{-1}, \quad \lambda(K)=\bigotimes_{i=0}^{m}\left(\lambda\left(K_{i}\right)\right)^{(-1)^{i}}, \\
& \tilde{\lambda}(K)=(\operatorname{det} H(X, K))^{-1}, \\
& \lambda(H(K))=\bigotimes_{i=0}^{m}\left(\operatorname{det} H\left(X, H^{i}(K)\right)^{(-1)^{i+1}} .\right.
\end{aligned}
$$


By [23, p. 46], the spectral sequence ' $E_{r}(K)$ (resp. " $\left.E_{r}(K)\right)$ induces the canonical functorial isomorphism:

$$
\lambda(K) \simeq \widetilde{\lambda}(K) \quad(\text { resp. } \lambda(H(K)) \simeq \widetilde{\lambda}(K)) .
$$

In our situation, $\xi=\left(\xi_{i}, v\right)$ is an orbifold resolution of the sheaf $i_{*} \mathcal{O}_{Y}(\eta)$.

Let $\mathcal{D}_{X}^{\bullet}$ be the sheaf of Dolbeault complex on $X$. For each $p \geq 0, \mathcal{D}_{X}^{p}$ is the sheaf of $\mathcal{C}^{\infty}$ sections of the vector bundle $\Lambda\left(T^{*(0,1)} X\right)$. The differential operator $\bar{\partial}^{X}$ is $\mathcal{O}_{X}$-linear. By using arguments as in [10, $\left.\left.\S 3 \mathrm{a}\right)\right],[26$, II $\left.\S 2 \mathrm{a})\right]$, we find that the double complex $\mathcal{D}_{X}^{\bullet}(\xi)=\left(\mathcal{O}_{X}(\xi) \otimes_{\mathcal{O}_{X}} \mathcal{D}_{X}^{\bullet}, \bar{\partial}^{X}, v\right)$ is an $F_{*}$-acyclic resolution of the complex $\left(\mathcal{O}_{X}(\xi), v\right)$.

Let $\mathrm{N}_{H}$ be the number operator of $\xi$, i.e. $\mathrm{N}_{H}$ acts on $\xi_{k}$ by multiplication by $k$. Let $\mathrm{N}_{V}^{X}, \mathrm{~N}_{V}^{Y}$ be the operators defining the $\mathbf{Z}$-grading on $\Lambda\left(T^{*(0,1)} X\right), \Lambda\left(T^{*(0,1)} Y\right)$.

Definition 3.3. Let $E$ (resp. $F$ ) be the set of smooth sections of $\Lambda\left(T^{*(0,1)} X\right) \widehat{\otimes} \xi$ on $X$ (resp. $\Lambda\left(T^{*(0,1)} Y\right) \otimes \eta$ on $Y$ ). $E$ is $\mathbf{Z}$-graded by the operator $\mathrm{N}_{V}^{X}-\mathrm{N}_{H}$, and $F$ is $\mathbf{Z}$-graded by the operator $\mathrm{N}_{V}^{Y}$. We also denote $E=E_{+} \oplus E_{-}, F=F_{+} \oplus F_{-}$ as the corresponding $\mathbf{Z}_{2}$-gradings of $E, F$.

If $\left.\alpha \in \Lambda\left(T^{*(0,1)} X\right)\right|_{Y},\left.f \in \xi_{k}\right|_{Y}$, set

$$
r(\alpha \widehat{\otimes} f)=\left\{\begin{array}{l}
0 \text { if } \quad k \neq 0, \\
i^{*} \alpha \otimes r f \quad \text { if } \quad k=0 .
\end{array}\right.
$$

In this case.

$$
{ }^{\prime \prime} E_{1}^{p, q}(\xi)=0 \quad \text { if } \quad q>0 ; \quad " \quad E_{1}^{p, 0}(\xi)=\Omega^{p}(X, \eta) .
$$

So " $E_{r}(\xi)$ degenerates for $r=2$, and " $E_{2}(\xi)$ is isomorphic to the hypercohomology of $\left(\mathcal{O}_{X}(\xi), v\right)$. By (3.8), we have

$$
{ }^{\prime \prime} E_{2}^{p, 0}(\xi)=H^{p}(X, \eta) .
$$

Thus we extend a result of [13, Theorem 1.7] to the orbifold case.

Theorem 3.4. The map $r:\left(E, \bar{\partial}^{X}+v\right) \rightarrow\left(F, \bar{\partial}^{Y}\right)$ is a quasi-isomorphism of $\mathbf{Z}$-graded complexes. It induces the canonical identification $H(X, \xi) \simeq H(Y, \eta)$.

Let $\rho, \tau$ be the canonical nonzero sections of the lines $\lambda^{-1}(\eta) \otimes \widetilde{\lambda}(\xi), \widetilde{\lambda}^{-1}(\xi) \otimes$ $\lambda(\xi)$ associated with the canonical isomorphism (3.6). Then the canonical nonzero section $\sigma$ of the line $\lambda^{-1}(\eta) \otimes \lambda(\xi)$ constructed as in [23, p. 46] is

$$
\sigma=\rho \otimes \tau .
$$

3.2. A Quillen metric on $\widetilde{\lambda}(\xi)$. Let $g^{T X}, h_{\xi}=\bigoplus h_{\xi_{i}}$ be smooth Hermitian metrics on $T X, \xi=\bigoplus \xi_{i}$. Let $g^{T Y}, h^{\eta}$ be metrics on $T Y, \eta$. By Section 2.2 these metrics induce Quillen metrics \|\|$_{\lambda\left(\xi_{i}\right)},\|\|_{\lambda(\eta)}$ on $\lambda\left(\xi_{i}\right), \lambda(\eta)$.

Let $v^{*}$ be the adjoint of $v$ with respect to $h^{\xi}$. Let $\bar{\partial}^{X *}$ be the formal adjoint of $\bar{\partial}^{X}$ with respect to the Hermitian product $\langle>$ on $E$ (2.8). Set

$$
\begin{aligned}
& D^{X}=\bar{\partial}^{X}+\bar{\partial}^{X *}, \quad V=v+v^{*}, \\
& \mathbf{K}=\left\{e \in E,\left(D^{X}+V\right) e=0\right\} .
\end{aligned}
$$

By Hodge theory, we have a canonical identification of $\mathbf{Z}$-graded vector spaces $\mathbf{K} \simeq H\left(E, \bar{\partial}^{X}+v\right)$. Let ||$_{\tilde{\lambda}(\xi)}$ be the metric on $\tilde{\lambda}(\xi)$ induced by the restriction 
of the $L_{2}$ metric \langle\rangle on $\mathbf{K} \simeq H\left(E, \bar{\partial}^{X}+v\right)$. Let $P$ be the orthogonal projection operator from $E$ on $\mathbf{K}$. Set $P^{\perp}=1-P$. For $s \in \mathbf{C}, \operatorname{Re}(s)>\operatorname{dim} X$, set

$$
\widetilde{\theta}_{\xi}^{X}(s)=-\operatorname{Tr}_{s}\left[\left(\mathrm{~N}_{V}^{X}-\mathrm{N}_{H}\right)\left(\left(D^{X}+V\right)^{2}\right)^{-s} P^{\perp}\right] .
$$

By (2.3), $\widetilde{\theta}_{\xi}^{X}(s)$ extends to a meromorphic function of $s \in \mathbf{C}$, which is holomorphic near $s=0$. Set

$$
\log \|\quad\|_{\tilde{\lambda}(\xi)}^{2}=\log ||_{\tilde{\lambda}(\xi)}^{2}-\frac{\partial \widetilde{\theta}_{\xi}^{X}}{\partial s}(0) .
$$

Tautologically, by (3.10), we get

$$
\|\sigma\|_{\lambda^{-1}(\eta) \otimes \lambda(\xi)}=\|\rho\|_{\lambda^{-1}(\eta) \otimes \tilde{\lambda}(\xi)} \mid\|\tau\|_{\tilde{\lambda}^{-1}(\xi) \otimes \lambda(\xi)} .
$$

By Theorem 0.1, to get a formula for (3.14) for arbitrary metrics $g^{T X}, h^{\xi_{0}}, \cdots$, $h^{\xi_{m}}, g^{T Y}, h^{\eta}$, with $g^{T X}, g^{T Y}$ Kähler, it is enough to calculate (3.14) for one choice of metrics.

\subsection{The norm of the section $\tau$.}

Theorem 3.5. The following identity holds:

$$
\log \|\tau\|_{\tilde{\lambda}^{-1}(\xi) \otimes \lambda(\xi)}^{2}=0
$$

Proof. The proof of (3.15) follows the same lines as the proof of 13 Theorem 2.1].

3.4. Assumption on the metrics on $T X, T Y, \xi, \eta$. Our first basic assumption is that the metric $g^{T X}$ is Kähler. Also we assume that $g^{T Y}$ is the metric induced by $g^{T X}$ on $T Y$. Let $\omega^{X}, \omega^{Y}$ be the Kähler forms of $\left(X, g^{T X}\right),\left(Y, g^{T Y}\right)$. Let $N$ be the normal bundle to $Y$ in $X$.

In the following, we assume that the metrics $h^{\xi_{0}}, \cdots, h^{\xi_{m}}$ verify assumption (A) of [3, §1b)], with respect to $g^{N}, h^{\eta}$. We describe this assumption in more detail.

On $Y$, we have the exact sequence of holomorphic orbifold vector bundles

$$
\left.0 \rightarrow T Y \rightarrow T X\right|_{Y} \rightarrow N \rightarrow 0
$$

We identify $N$ to the orthogonal orbifold bundle to $T Y$ in $\left.T X\right|_{Y}$. Let $g^{N}$ be the metric induced by $g^{\left.T X\right|_{Y}}$ on $N$.

By the argument as in [7, §3d)], 3, §1b)], we have:

For $y \in Y$, let $H_{y}(\xi, v)$ be the homology of the complex $(\xi, v)_{y}$. If $y \in Y, u \in T_{y} X$, let $\partial_{u} v(y)$ be the derivative of $v$ at $y$ in the direction $u$ in any given holomorphic trivialization of $(\xi, v)$ near $y$. Then

a) The $H_{y}(\xi, v)$ are the fibers of a holomorphic $\mathbf{Z}$-graded vector orbifold bundle $H(\xi, v)$ on $Y$. The map $\partial_{u} v(y)$ acts on $H(\xi, v)_{y}$ as a chain map. This action does not depend on the trivialization of $(\xi, v)$, but only depends on the image $z$ of $u$ in $N_{y}$. From now on, we will write $\partial_{z} v(y)$ instead of $\partial_{u} v(y)$.

b) Let $\pi$ be the projection $N \rightarrow Y$. Then over $N$, we have a canonical identification of $\mathbf{Z}$-graded chain complexes of orbifold bundles

$$
\left(\pi^{*} H(\xi, v), \partial_{z} v(y)\right) \simeq\left(\pi^{*}\left(\Lambda\left(N^{*}\right) \otimes \eta\right), \sqrt{-1} i_{z}\right) .
$$

By finite dimensional Hodge theory, we know that for $V \in \mathcal{V}, V \subset X$, there is a canonical isomorphism of $\mathbf{Z}$-graded vector spaces over $\widetilde{V}$

$$
\widetilde{H}(\xi, v) \simeq\left\{f \in \widetilde{\xi}, \quad v f=0, \quad v^{*} f=0\right\} .
$$


Let $h^{H(\xi, v)}$ be the induced metric on $H(\xi, v)$ by the identification (3.18).

Let $h^{\Lambda\left(N^{*}\right) \otimes \eta}$ be the metric induced by $g^{N}$ and $h^{\eta}$ on $\Lambda\left(N^{*}\right) \otimes \eta$.

Definition 3.6. We say that the metrics $h^{\xi_{0}}, \cdots, h^{\xi_{m}}$ verify assumption (A) with respect to $g^{N}, h^{\eta}$, if the identification (3.17) also identifies the metrics.

Proposition 3.7. There exist metrics $h^{\xi_{0}}, \cdots, h^{\xi_{m}}$ on $\xi_{0}, \cdots, \xi_{m}$ which verify assumption (A) with respect to $g^{N}, h^{\eta}$.

Proof. We first choose arbitrary smooth Hermitian metrics $\left.h^{\prime \xi_{i}}\right|_{Y}$ on $\left.\xi_{i}\right|_{Y}$. Then on each local chart $\left(G_{U}^{\xi}, \widetilde{U}\right)$, we will choose a $G_{U}^{\xi}$-invariant metric in the procedure of the proof of [3, Proposition 1.6]. Lastly, we get the result.

\section{A singular Bott-Chern current}

In this section, we construct Bott-Chern currents associated to the Hermitian chain complex $\left((\xi, v), h^{\xi}\right)$. We extend [11] to the orbifold setting.

In this section, we use the same assumption and the same notation as in Section 3.4.

Let $U \subset Y, V \subset X, U=V \cap Y$, be open subsets as in Definition 1.6. For $g \in G_{V}$, let $K_{\widetilde{V}^{g}}=\operatorname{Ker}\left\{Z_{G_{V}}(g) \rightarrow \operatorname{Diffeo}\left(\widetilde{V}^{g}\right)\right\}, K_{\widetilde{U}^{g}}=\operatorname{Ker}\left\{Z_{G_{V}}(g) \rightarrow \operatorname{Diffeo}\left(\widetilde{U}^{g}\right)\right\}$.

Let $\delta_{Y}$ be the current on $X$ defined by: If $\varphi$ is a form on $X, \operatorname{supp} \varphi \subset V$, then

$$
\int_{X} \delta_{Y} \varphi=\left.\frac{1}{\left|G_{V}\right|} \int_{\widetilde{U}} \varphi\right|_{\widetilde{U}}=\left.\frac{1}{\left|G_{V} / K_{U}\right|} \int_{Y} \varphi\right|_{Y}
$$

Let $X_{i}$ be a connected component of $\Sigma X$. If $X_{i}$ is represented by $\widetilde{V}^{g}\left(g \in G_{V}\right)$ over $\widetilde{V}$, then

$$
\left(Z_{G_{V}}(g) / K_{\widetilde{U}^{g}}, \widetilde{U}^{g}\right) \stackrel{\pi}{\rightarrow} \widetilde{U}^{g} / Z_{G_{V}}(g)
$$

also defines an orbifold structure. We denote it by $Y^{\prime}$. Then $Y \cup Y^{\prime}$ is a sub-orbifold of $X \cup \Sigma X$. Set

$$
m_{X, Y}=\left|K_{\widetilde{U}^{g}}\right| /\left|K_{\widetilde{V}^{g}}\right| .
$$

Then $m_{X, Y}$ is a local constant on $Y \cup Y^{\prime}$. The number $m_{X, Y}$ is the relative multiplicity of $Y \cup Y^{\prime}$ in $X \cup \Sigma X$ in Definition 1.6

Let $\nabla^{\xi}=\bigoplus_{i=0}^{m} \nabla^{\xi_{i}}$ be the holomorphic Hermitian connection on $\left(\xi, h^{\xi}\right)=$ $\bigoplus_{i=0}^{m}\left(\xi_{i}, h^{\xi_{i}}\right)$. Clearly $V=v+v^{*}$ is a self-adjoint section of $\operatorname{End}^{\text {odd }}(\xi)$. For $u>0$, set

$$
C_{u}=\nabla^{\xi}+\sqrt{u} V
$$

Then $C_{u}$ is a $G_{V}$-invariant superconnection on the $\mathbf{Z}_{2^{-}}$graded vector bundle $\xi$ on $\widetilde{V}$.

Let $\Phi$ be the homomorphism of $\Lambda^{\text {even }}\left(T_{\mathbf{R}}^{*} X\right)$ into itself: $\alpha \rightarrow(2 i \pi)^{-\operatorname{deg} \alpha / 2} \alpha$.

We define the forms $\operatorname{ch}^{\Sigma}\left(\eta, h^{\eta}\right),\left(\operatorname{Td}^{\Sigma}\right)^{-1}\left(N, g^{N}\right)$ over $Y \cup Y^{\prime}$ by $\operatorname{ch}_{g}\left(\eta, h^{\eta}\right)$, $\left(\operatorname{Td}_{g}\right)^{-1}\left(N, g^{N}\right)$ on $\widetilde{U}^{g} / Z_{G_{V}}(g)$. Then we construct forms $\operatorname{Tir}_{s}^{\Sigma}\left[\exp \left(-C_{u}^{2}\right)\right]$, $\Phi \operatorname{Tr}_{s}^{\Sigma}\left[\mathrm{N}_{H} \exp \left(-C_{u}^{2}\right)\right]$ and $T^{\Sigma}\left(\xi, h^{\xi}\right)$ on $X \cup \Sigma X$ : over $\widetilde{V}^{g} / Z_{G_{V}}(g)$, they are defined by $\Phi \operatorname{Tr}_{s}\left[g \exp \left(-C_{u}^{2}\right)\right], \Phi \operatorname{Tr}_{s}\left[\mathrm{~N}_{H} g \exp \left(-C_{u}^{2}\right)\right]$ and $T_{g}\left(\xi, h^{\xi}\right)$ (cf. [6. Definition 6.6]). Then by [6, Theorem 6.7], $T^{\Sigma}\left(\xi, h^{\xi}\right) \in P^{X \cup \Sigma X}$ such that

$$
\frac{\bar{\partial} \partial}{2 i \pi} T^{\Sigma}\left(\xi, h^{\xi}\right)=\left(\operatorname{Td}^{\Sigma}\right)^{-1}\left(N, g^{N}\right) \operatorname{ch}^{\Sigma}\left(\eta, h^{\eta}\right) \delta_{Y \cup Y^{\prime}}-\operatorname{ch}^{\Sigma}\left(\xi, h^{\xi}\right) .
$$




\section{The AnAlytic torsion forms of A Short EXACT ORBIFOld BUndLE}

In this section, using the construction in [5] of analytic torsion forms, we extend the construction of [4] to an orbifold situation.

This section is organized as follows. In Section 5.1 we extend the analytic torsion forms in [5, §6] to orbifolds. In Section 5.2, using the result of [5], we evaluate these forms in terms of Bott-Chern classes.

In this section, we use the notation of Section 1.1.

5.1. Generalized analytic torsion forms. Let $(B, \mathcal{U})$ be a compact complex orbifold. Let

$$
E: 0 \rightarrow L \rightarrow M \rightarrow N \rightarrow 0
$$

be a short exact sequence of holomorphic orbifold vector bundles on $B$ (cf. Definition 1.10). Let $h^{M}$ be a Hermitian metric on $M$. Let $h^{L}, h^{N}$ be the induced metrics on $L, N$.

For a local chart $\left(G_{U}, \widetilde{U}\right) \rightarrow U$, let $\gamma_{U}: G_{U}^{E} \rightarrow G_{U}=G_{U}^{E} / K_{U}^{E}$ be the natural map. In the sequence, we will work on $\widetilde{U}$, and we will omit the subscript $U$.

Let $d v_{\widetilde{M}}, d v_{\widetilde{N}}$ be the Riemannian volume forms on the fibers $\widetilde{M}_{\mathbf{R}}, \widetilde{N}_{\mathbf{R}}$. The smooth kernels on the fibers of $\widetilde{M}_{\mathbf{R}}$ will be calculated with respect to $d v_{\widetilde{M}} /(2 \pi)^{\operatorname{dim} M}$.

Let $\mathcal{B}_{u}^{2}$ be the differential operator on $\widetilde{M}_{U}$, defined by [4, Theorem 3.10], [6], Definition 7.4]. For $y \in \widetilde{U}, u>0$, let $Q_{u}^{y}\left(z, z^{\prime}\right)\left(z, z^{\prime} \in \widetilde{M}_{\mathbf{R}, y}\right)$ be the smooth kernel associated to $\exp \left(-\mathcal{B}_{u}^{2}\right)$. For the existence and the uniqueness of $Q_{u}^{y}\left(z, z^{\prime}\right)$, we refer to 4 [ $§ 4 \mathrm{a})$ ].

Tautologically for each $g \in G_{U}^{E}, g$ is an isometric of $\widetilde{M}$ on $\widetilde{U}^{g}$, which is parallel with respect to $\nabla^{\widetilde{M}}$. Let $e^{i \theta_{1, g}}, \cdots, e^{i \theta_{q, g}}\left(0 \leq \theta_{j, g}<2 \pi\right)$ be the locally constant distinct eigenvalues of $g \in G_{U}^{E}$ acting on $\widetilde{L}, \widetilde{M}, \widetilde{N}$ on $\widetilde{U}^{g}$. Then $\widetilde{E}$ splits holomorphically as an orthogonal sum of complexes

$$
E^{\theta_{j, g}}: 0 \rightarrow \widetilde{L}^{\theta_{j, g}} \rightarrow \widetilde{M}^{\theta_{j, g}} \rightarrow \widetilde{N}^{\theta_{j, g}} \rightarrow 0
$$

and $g$ acts on $E^{\theta_{j, g}}$ by multiplication by $e^{i \theta_{j, g}}$. Set $\widetilde{L}^{0, \perp, g}=\bigoplus_{\theta_{j, g} \neq 0} \widetilde{L}^{\theta_{j, g}}$, etc.

Clearly $g$ acts on $\Lambda\left(\overline{\widetilde{L}}^{0, \perp, g, *}\right) \widehat{\otimes} \Lambda\left(\overline{\widetilde{N}}^{*}\right) \widehat{\otimes} \Lambda\left(\widetilde{N}^{*}\right)$ and so $g$ acts on

$$
\Lambda\left(T_{\mathbf{R}}^{*} \widetilde{B}\right) \widehat{\otimes} \Lambda\left(\overline{\widetilde{L}}^{0, \perp, g, *}\right) \widehat{\otimes} \Lambda\left(\overline{\widetilde{N}}^{*}\right) \widehat{\otimes} \Lambda\left(\widetilde{N}^{*}\right) .
$$

Also $Q_{u}^{y}\left(z, z^{\prime}\right)$ acts on the same bundle (it acts trivially on $\left.\Lambda\left(\widetilde{\widetilde{L}}^{0, \perp, g, *}\right)\right)$. Therefore

$$
g Q_{u}^{y}\left(g^{-1} z, z^{\prime}\right) \in\left(\Lambda\left(T_{\mathbf{R}}^{*} \widetilde{B}\right) \widehat{\otimes} \operatorname{End}\left(\Lambda\left(\widetilde{\widetilde{L}}^{0, \perp, g, *}\right) \widehat{\otimes} \Lambda\left(\overline{\widetilde{N}}^{*}\right) \widehat{\otimes} \Lambda\left(\widetilde{N}^{*}\right)\right)\right)^{\text {even }} .
$$

Let $\operatorname{Tr}_{s}\left[g Q_{u}^{y}\left(g^{-1} z, z\right)\right] \in \Lambda\left(T_{\mathbf{R}}^{*} \widetilde{B}\right)$ denote the corresponding supertrace. Let $\mathrm{N}_{H}$ be the number operator of $\Lambda\left(N^{*}\right)$. Then $\mathrm{N}_{H}$ acts like $1 \widehat{\otimes} \mathrm{N}_{H}$ on $\Lambda\left(T_{\mathbf{R}}^{*} \widetilde{B}\right) \widehat{\otimes} \Lambda\left(\overline{\widetilde{L}}^{0, \perp, g, *}\right)$ $\widehat{\otimes} \Lambda\left(\overline{\widetilde{N}}^{*}\right) \widehat{\otimes} \Lambda\left(\tilde{N}^{*}\right)$.

Let $B^{\prime}$ be the orbifold defined by: for $g \in G_{U}^{E}$, the local chart is $\left(Z_{G_{U}^{E}}(g), \widetilde{U}^{g}\right) \rightarrow$ $\widetilde{U}^{g} / Z_{G_{U}^{E}}(g)$. 
Definition 5.1. For $u>0, g \in G_{U}^{E}$, over $\widetilde{U}^{g} / Z_{G_{U}^{E}}(g)$, set

$$
\begin{aligned}
& \operatorname{Tr}_{s}^{\Sigma}\left[\exp \left(-\mathcal{B}_{u}^{2}\right)\right]=\int_{\widetilde{M}_{\mathbf{R}}^{0, \perp, g} \oplus \widetilde{N}_{\mathbf{R}}^{0, g}} \operatorname{Tr}_{s}\left[g Q_{u}^{y}\left(g^{-1} z, z\right)\right] \frac{d v_{\widetilde{M}^{0, \perp, g} \oplus \widetilde{N}^{0, g}}(z)}{(2 \pi)^{\operatorname{dim} M^{0, \perp, g}+\operatorname{dim} N^{0, g}}}, \\
& \operatorname{Tr}_{s}^{\Sigma}\left[\mathrm{N}_{H} \exp \left(-\mathcal{B}_{u}^{2}\right)\right]=\int_{\widetilde{M}_{\mathbf{R}}^{0, \perp, g} \oplus \widetilde{N}_{\mathbf{R}}^{0, g}} \operatorname{Tr}_{s}\left[\mathrm{~N}_{H} g Q_{u}^{y}\left(g^{-1} z, z\right)\right] \frac{d v_{\widetilde{M}^{0, \perp, g} \oplus \widetilde{N}^{0, g}}(z)}{(2 \pi)^{\operatorname{dim} M^{0, \perp, g}+\operatorname{dim} N^{0, g}}} .
\end{aligned}
$$

The objects constructed in (5.3) are smooth forms on $B \cup B^{\prime}$.

Now we reproduce the construction given in [5, $\S 6]$ of analytic torsion forms.

Definition 5.2. For $s \in \mathbf{C}, 0<\operatorname{Re}(s)<\frac{1}{2}$, let $A(s)$ be the form on $B \cup B^{\prime}$,

$$
A(s)=\frac{1}{\Gamma(s)} \int_{0}^{+\infty} u^{s-1}\left\{\Phi \operatorname{Tr}_{s}^{\Sigma}\left[\mathrm{N}_{H} \exp \left(-\mathcal{B}_{u}^{2}\right)\right]-\frac{\operatorname{dim} N}{2} \operatorname{Td}^{\Sigma}\left(L, h^{L}\right)\right\} d u .
$$

By [6, Theorem 7.6], one verifies that $s \rightarrow A(s)$ extends to a function which is holomorphic near $s=0$. Set

$$
B\left(L, M, h^{M}\right)=\frac{\partial A}{\partial s}(0) .
$$

As in Section 1.2 we define the form $\mathrm{Td}^{\Sigma}, \mathrm{ch}^{\Sigma}$ on $B \cup B^{\prime}$. By proceeding as in [5, Theorem 6.3], we have

Theorem 5.3. The form $B\left(L, M, h^{M}\right)$ lies in $P^{B \cup B^{\prime}}$. Moreover

$$
\frac{\bar{\partial} \partial}{2 i \pi} B\left(L, M, h^{M}\right)=\operatorname{Td}^{\Sigma}\left(L, h^{L}\right)-\frac{\operatorname{Td}^{\Sigma}\left(M, h^{M}\right)}{\operatorname{Td}^{\Sigma}\left(N, h^{N}\right)} .
$$

5.2. Evaluation of the generalized analytic torsion forms. Recall that the Hirzebruch polynomial $\widehat{A}(x)$ is given by $\widehat{A}(x)=\frac{x / 2}{\sinh (x / 2)}$. Set

$$
\alpha(\theta, x)=\left\{\begin{array}{l}
\widehat{A}(x) \quad \text { if } \quad \theta \in 2 \pi \mathbf{Z}, \\
\frac{\widehat{A}(x+i \theta)}{x+i \theta} \quad \text { if } \quad \theta \in \mathbf{R}-2 \pi \mathbf{Z} .
\end{array}\right.
$$

Let $D(\theta, x)(\theta \in \mathbf{R}$, and $x \in \mathbf{C})$ be the function defined in [5] Definition 6.5]. For $\theta \in \mathbf{R}$, we identify $D(\theta, x)$ with the corresponding additive genus. Let $D^{\Sigma}\left(N, h^{N}\right)$ be the form on $B \cup B^{\prime}$ associated to $D_{g}$ in [5, Definition 6.7].

Definition 5.4. For $\theta \in \mathbf{R}, x \in \mathbf{C},|x|<2 \pi$ if $\theta \in 2 \pi \mathbf{Z}$; $|x|<\inf _{k \in \mathbf{Z}}|\theta+2 k \pi|$ if $\theta \in \mathbf{R}-2 \pi \mathbf{Z}$, set

$$
R(\theta, x)=D(\theta, x)-\Gamma^{\prime}(1) \frac{\partial \alpha / \partial x}{\alpha}(\theta, x) .
$$

As above, we denote by $R^{\Sigma}$ the corresponding additive genus.

Let $\widetilde{\operatorname{Td}}^{\Sigma}\left(L, M, h^{M}\right) \in P^{B \cup B^{\prime}} / P^{B \cup B^{\prime}, 0}$ be the Bott-Chern class constructed as in Section [1.2, [8 $\S 1 \mathrm{f})]$, such that

$$
\frac{\bar{\partial} \partial}{2 i \pi} \widetilde{\operatorname{Td}}^{\Sigma}\left(L, M, h^{M}\right)=\operatorname{Td}^{\Sigma}\left(M, h^{M}\right)-\operatorname{Td}^{\Sigma}\left(L, h^{L}\right) \operatorname{Td}^{\Sigma}\left(N, h^{N}\right) .
$$

Now the computation in [5. Theorem 6.8] is local and universal, thus we can work on each $\widetilde{U}$ and we get the following extension of [5] Theorem 6.8]. 
Theorem 5.5. The following identity holds in $P^{B \cup B^{\prime}} / P^{B \cup B^{\prime}, 0}$ :

$$
B\left(L, M, h^{M}\right)=-\left(\mathrm{Td}^{\Sigma}\right)^{-1}\left(N, h^{N}\right) \widetilde{\mathrm{Td}}^{\Sigma}\left(L, M, h^{M}\right)+\operatorname{Td}^{\Sigma}\left(L, h^{L}\right) D^{\Sigma}\left(N, h^{N}\right) .
$$

\section{IMMERSIONS AND QUILLEN METRICS}

In this section, we prove Theorem 0.2 which calculates the Quillen norm of the canonical section $\sigma$ of $\lambda^{-1}(\eta) \otimes \lambda(\xi)$. This extends the result of 13 , Theorem $0.1]$ to the orbifold case. Let $Y^{\prime}$ be the sub-orbifold of $\Sigma X$ constructed as in Section [4 If we compare it with the formula [13, (6.2)], we guess naturally that Theorem 0.2 will contain some integral on $Y \cup \Sigma Y$. In fact, we use the integral over $Y \cup Y^{\prime}$.

This section is organized as follows. In Section 6.1, we introduce a closed 1-form. In Section 6.2, we state seven intermediate results which we need for the proof of Theorem 0.2, whose proofs are delayed to Sections 6.4.6.8 In Section 6.3, we prove Theorem 0.2. In Section 6.4 we prove Theorems 6.3, 6.4 In Section 6.5, we prove Theorem 6.8 In Section 6.6, we prove Theorem 6.5. In Section 6.7, we prove Theorem 6.6. In Section 6.8 we prove Theorem 6.7.

In this section, we use the same assumption as in Section [3. We use also the notation of Sections 13.5.

6.1. A closed 1-form on $\mathbf{R}_{+}^{*} \times \mathbf{R}_{+}^{*}$. Let $i: Y \rightarrow X$ be a holomorphic orbifold immersion of compact complex orbifolds. Let $\eta$ be a holomorphic orbifold vector bundle on $Y$. Let $(\xi, v)$ be an orbifold resolution of holomorphic vector bundles of the sheaf $i_{*} \mathcal{O}_{Y}(\eta)$. By (3.10), there is a nonzero canonical section $\sigma$ of $\lambda^{-1}(\eta) \otimes \lambda(\xi)$. The operators $D^{X}, V$ were defined in (3.11). For $u>0, T>0$, set

$$
B_{u, T}=u\left(D^{X}+T V\right), \quad A_{T}=D^{X}+T V .
$$

By proceeding as in [13, Theorem 3.5], we have

Theorem 6.1. Let $\beta_{u, T}$ be the 1-form on $\mathbf{R}_{+}^{*} \times \mathbf{R}_{+}^{*}$,

$$
\beta_{u, T}=\frac{d u}{u} \operatorname{Tr}_{s}\left[\left(\mathrm{~N}_{V}^{X}-\mathrm{N}_{H}\right) \exp \left(-B_{u, T}^{2}\right)\right]-\frac{d T}{T} \operatorname{Tr}_{s}\left[\mathrm{~N}_{H} \exp \left(-B_{u, T}^{2}\right)\right] .
$$

Then $\beta_{u, T}$ is closed.

Take $\epsilon, A, T\left(0<\epsilon \leq 1 \leq A<+\infty, 1 \leq T_{0}<+\infty\right)$. Let $\Gamma=\bigcup_{i=1}^{4} \Gamma_{i}$ be the oriented contour in $\mathbf{R}_{+}^{*} \times \mathbf{R}_{+}^{*}$ :

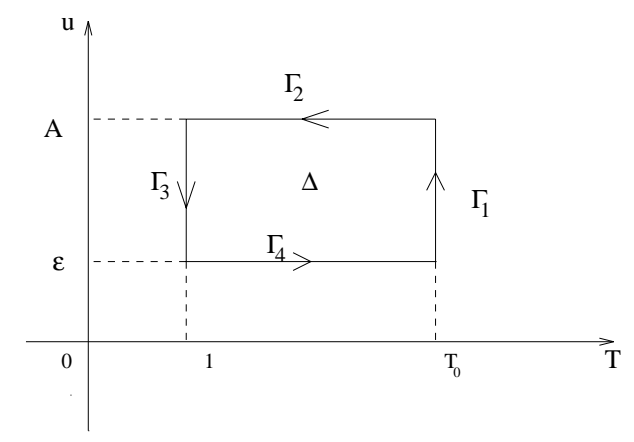


For $1 \leq k \leq 4$, set $I_{k}^{0}=\int_{\Gamma_{k}} \beta_{u, T}$. From Theorem 6.1, we get

$$
\sum_{k=1}^{4} I_{k}^{0}=0 .
$$

6.2. Seven intermediate results. Let $\bar{\partial}^{Y *}$ be the formal adjoint of $\bar{\partial}^{Y}$. Set $D^{Y}=\bar{\partial}^{Y}+\bar{\partial}^{Y *}$.

Let $Y^{\prime}$ be the sub-orbifold of $\Sigma X$ constructed in Section 4. Let $m_{i, X}$ be the multiplicity of the connected component $X_{i}$ of $X \cup \Sigma X$. The relative multiplicity $m_{X_{i}, Y_{j}^{\prime}}$ on $Y_{j}^{\prime} \subset X_{i}$ is defined by (4.2); then $m_{X_{i}, Y_{j}^{\prime}}$ is constant on $Y_{j}^{\prime}$.

Recall that $\omega^{X}, \omega^{Y}$ are the Kähler forms of $X, Y$. Since these forms are closed, they can be paired with characteristic classes of vector bundles on $\Sigma X, \Sigma Y$, respectively.

Let $m_{l, Y^{j}}$ be the multiplicity of the connected component $Y_{l}^{j}$ of $Y^{j} \cup \Sigma Y^{j}$. For $0 \leq i \leq m, 1 \leq j \leq d$, let $\chi\left(\xi_{i}\right), \chi\left(\eta^{j}\right)$ be the Euler characteristics of $\xi_{i}, \eta^{j}$. By Kawasaki-Riemann-Roch Theorem [21,

$$
\begin{aligned}
& \chi\left(\xi_{i}\right)=\sum_{j} \frac{1}{m_{j, X}} \int_{X_{j}} \operatorname{Td}^{\Sigma}(T X) \operatorname{ch}^{\Sigma}\left(\xi_{i}\right), \\
& \chi\left(\eta^{j}\right)=\sum_{l} \frac{1}{m_{l, Y^{j}}} \int_{Y_{l}^{j}} \operatorname{Td}^{\Sigma}(T Y) \operatorname{ch}^{\Sigma}(\eta) .
\end{aligned}
$$

In what follows, we will often use the notation

$$
\begin{aligned}
& \operatorname{ch}^{\Sigma}(\xi)=\sum_{i=0}^{m}(-1)^{i} \operatorname{ch}^{\Sigma}\left(\xi_{i}\right), \quad \operatorname{ch}^{\prime \Sigma}(\xi)=\sum_{i=0}^{m}(-1)^{i} i \operatorname{ch}^{\Sigma}\left(\xi_{i}\right), \\
& \operatorname{dim} N \chi(\eta)=\sum_{j} \operatorname{dim} N_{j} \chi\left(\eta^{j}\right) .
\end{aligned}
$$

We now state seven intermediate results contained in Theorems 6.26 .8

Theorem 6.2. As $u \rightarrow 0$,

$$
\begin{aligned}
& \operatorname{Tr}_{s}\left[\left(\mathrm{~N}_{V}^{X}-\mathrm{N}_{H}\right) \exp \left(-u\left(D^{X}+V\right)^{2}\right)\right]=\sum_{i} \frac{1}{u m_{i, X}} \int_{X_{i}} \frac{\omega^{X}}{2 \pi} \operatorname{Td}^{\Sigma}(T X) \operatorname{ch}^{\Sigma}(\xi) \\
& +\sum_{i} \frac{1}{m_{i, X}} \int_{X_{i}}\left[\left(\operatorname{dim} X \mathrm{Td}^{\Sigma}(T X)-\mathrm{Td}^{\prime \Sigma}(T X)\right) \operatorname{ch}^{\Sigma}(\xi)\right. \\
& \left.-\mathrm{Td}^{\Sigma}(T X) \operatorname{ch}^{\prime \Sigma}(\xi)\right]+O(u), \\
& \operatorname{Tr}_{s}\left[\mathrm{~N}_{V}^{Y} \exp \left(-u D^{Y, 2}\right)\right]=\sum_{l, j} \frac{1}{m_{l, Y^{j}}}\left[\frac{1}{u} \int_{Y_{l}^{j}} \frac{\omega^{Y}}{2 \pi} \operatorname{Td}^{\Sigma}\left(T Y^{j}\right) \operatorname{ch}^{\Sigma}\left(\eta^{j}\right)\right. \\
& \left.+\int_{Y_{l}^{j}}\left(\operatorname{dim} Y^{j} \operatorname{Td}^{\Sigma}\left(T Y^{j}\right)-\operatorname{Td}^{\Sigma}\left(T Y^{j}\right)\right) \operatorname{ch}^{\Sigma}\left(\eta^{j}\right)\right]+O(u) .
\end{aligned}
$$

Proof. We get the second identity from (2.25). The proof of the first identity follows the same line. 
Theorem 6.3. For any $u_{0}>0$, there exists $C>0$ such that for $u \geq u_{0}, T \geq 1$,

$$
\begin{aligned}
& \left|\operatorname{Tr}_{s}\left[\left(\mathrm{~N}_{V}^{X}-\mathrm{N}_{H}\right) \exp \left(-u\left(D^{X}+T V\right)^{2}\right)\right]-\operatorname{Tr}_{s}\left[\mathrm{~N}_{V}^{Y} \exp \left(-u D^{Y, 2}\right)\right]\right| \leq \frac{C}{\sqrt{T}}, \\
& \left|\operatorname{Tr}_{s}\left[\mathrm{~N}_{H} \exp \left(-u\left(D^{X}+T V\right)^{2}\right)\right]-\frac{1}{2} \operatorname{dim} N \chi(\eta)\right| \leq \frac{C}{\sqrt{T}} .
\end{aligned}
$$

Theorem 6.4. There exist $c>0, C>0$ such that for $u \geq 1, T \geq 1$,

$$
\left|\operatorname{Tr}_{s}\left[\left(\mathrm{~N}_{V}^{X}-\mathrm{N}_{H}\right) \exp \left(-u\left(D^{X}+T V\right)^{2}\right)\right]-\operatorname{Tr}_{s}\left[\left(\mathrm{~N}_{V}^{X}-\mathrm{N}_{H}\right) \widetilde{P}_{T}\right]\right| \leq c e^{-C u} .
$$

Here $\widetilde{P}_{T}$ is the orthogonal projection form $E$ on $\operatorname{Ker}\left(D^{X}+T V\right)$ with respect to the Hermitian product on $E$ induced by $g^{T X}, h^{\xi}$ (cf. (2.8)).

Theorem 6.5. There exist $C>0, \gamma \in] 0,1]$ such that for $u \in] 0,1], 0 \leq T \leq 1 / u$,

$$
\begin{aligned}
& \mid \operatorname{Tr}_{s}\left[\mathrm{~N}_{H} \exp \left(-\left(u D^{X}+T V\right)^{2}\right)\right] \\
& \quad-\sum_{i} \frac{1}{m_{i, X}} \int_{X_{i}} \operatorname{Td}^{\Sigma}\left(T X, g^{T X}\right) \Phi \operatorname{Tr}_{s}^{\Sigma}\left[\mathrm{N}_{H} \exp \left(-C_{T^{2}}^{2}\right)\right] \mid \leq C(u(1+T))^{\gamma} .
\end{aligned}
$$

There exists $C^{\prime}>0$ such that for $\left.\left.u \in\right] 0,1\right], 0 \leq T \leq 1$,

$$
\left|\operatorname{Tr}_{s}\left[\mathrm{~N}_{H} \exp \left(-\left(u D^{X}+T V\right)^{2}\right)\right]-\operatorname{Tr}_{s}\left[\mathrm{~N}_{H} \exp \left(-\left(u D^{X}\right)^{2}\right)\right]\right| \leq C^{\prime} T
$$

In the following, we use the notation of Section 5 applied to the exact sequence of holomorphic Hermitian orbifold vector bundles on $Y:\left.0 \rightarrow T Y \rightarrow T X\right|_{Y} \rightarrow N \rightarrow 0$. In particular, for $u>0$, we consider the operator $\mathcal{B}_{u}^{2}$ in Section 5.1 .

Theorem 6.6. For any $T>0$, the following identity holds:

$$
\begin{aligned}
\lim _{u \rightarrow 0} & \operatorname{Tr}_{s}\left[\mathrm{~N}_{H} \exp \left(-\left(u D^{X}+\frac{T}{u} V\right)^{2}\right)\right] \\
& =\sum_{i, j ; Y_{j}^{\prime} \subset X_{i}} \frac{1}{m_{i, X} m_{X_{i}, Y_{j}^{\prime}}} \int_{Y_{j}^{\prime}} \Phi \operatorname{Tr}_{s}^{\Sigma}\left[\mathrm{N}_{H} \exp \left(-\mathcal{B}_{T^{2}}^{2}\right)\right] \operatorname{ch}^{\Sigma}\left(\eta, h^{\eta}\right) .
\end{aligned}
$$

Theorem 6.7. There exist $C>0, \delta \in[0,1]$, such that for $u \in] 0,1], T \geq 1$,

$$
\left|\operatorname{Tr}_{s}\left[\mathrm{~N}_{H} \exp \left(-\left(u D^{X}+\frac{T}{u} V\right)^{2}\right)\right]-\frac{1}{2} \operatorname{dim} N \chi(\eta)\right| \leq \frac{C}{T^{\delta}} .
$$

Let $|\quad|_{\tilde{\lambda}(\xi), T}^{2}$ be the metric on $\tilde{\lambda}(\xi)$ inherited from the metrics $g^{T X}, \oplus_{i=0}^{m} T^{-2 i} h^{\xi_{i}}$ as in Section 3.2

Theorem 6.8. As $T \rightarrow+\infty$,

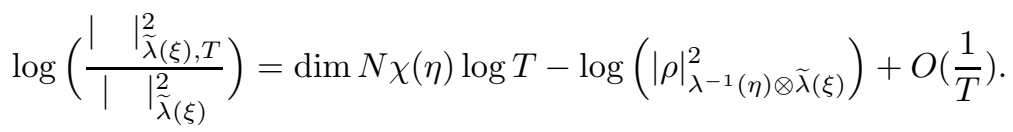

6.3. Proof of Theorem $\mathbf{0 . 2}$ At a formal level, Theorems 6.26.8 can be obtained from [13, Theorems 6.3-6.9], [6, Theorems 8.3-8.9]. This permits us to transfer the discussion in [13, §6] to our situation. Using Theorem [3.5] as in [13] §6], we get Theorem 0.2 . 
Remark 6.9. By (4.1) and (4.4),

$$
\begin{aligned}
\sum_{i, j ; Y_{j}^{\prime} \subset X_{i}} \frac{1}{m_{i, X} m_{X_{i}, Y_{j}^{\prime}}} \int_{Y_{j}^{\prime}} \operatorname{Td}^{\Sigma}(T X)\left(R^{\Sigma}(N)+R^{\Sigma}(T Y)\right) \operatorname{ch}^{\Sigma}(\eta) \\
=\sum_{i} \frac{1}{m_{i, X}} \int_{X_{i}} \operatorname{Td}^{\Sigma}(T X) R^{\Sigma}(T X) \operatorname{ch}^{\Sigma}(\xi) .
\end{aligned}
$$

Thus we have a similar formula as in [13, $\left.\left(6.1^{\prime}\right)\right]$.

6.4. Proof of Theorems 6.3 and 6.4. In this part, we give a proof of Theorems 6.3 and 6.4. This proof relies essentially on the results of [13, $\S 8$ and $\S 9]$, where the corresponding results were established when $X, Y$ are manifolds. We will use the same notation as in $[13, \S 8$ and $\S 9]$.

Recall that the orbifold normal bundle $N$ on $Y$ is identified with the orthogonal bundle to $T Y$ in $\left.T X\right|_{Y}$. We have the identification of $\mathcal{C}^{\infty}$ orbifold vector bundles on $Y,\left.T X\right|_{Y}=T Y \oplus N$. Thus we deduce the identification of $\mathcal{C}^{\infty}$ orbifold vector bundles on $Y$

$$
\left.\Lambda\left(T^{*(0,1)} X\right)\right|_{Y}=\Lambda\left(T^{*(0,1)} Y\right) \widehat{\otimes} \Lambda\left(\bar{N}^{*}\right) .
$$

By (3.17) and (6.15), we find that $\Lambda\left(\bar{N}^{*}\right) \widehat{\otimes} \Lambda\left(N^{*}\right) \otimes \eta$ is now an orbifold vector sub-bundle of $\left.\left(\Lambda\left(T^{*(0,1)} X\right) \widehat{\otimes} \xi\right)\right|_{Y}$.

For $y \in Y$, let $\theta_{y}$ denote the Kähler form of the fiber $N_{\mathbf{R}, y}$; then $\theta \in \Lambda^{1}\left(\bar{N}^{*}\right) \widehat{\otimes}$ $\Lambda^{1}\left(N^{*}\right)$. Let $\varphi$ denote the linear map

$$
\varphi:\left.a \in \Lambda\left(T^{*(0,1)} Y\right) \otimes \eta \rightarrow \frac{a \exp (\theta)}{2^{(\operatorname{dim} N) / 2}} \in\left(\Lambda\left(T^{*(0,1)} X\right) \widehat{\otimes} \xi\right)\right|_{Y} .
$$

As in [13, (7.14)], we find that $\varphi$ is norm preserving. Let $q$ be the orthogonal projection from $\left.\left(\Lambda\left(T^{*(0,1)} X\right) \widehat{\otimes} \xi\right)\right|_{Y}$ on the image of $\varphi$.

By Definition [1.6, over every local coordinate system $\left(G_{V}, \widetilde{V}\right) \stackrel{\tau_{V}}{\rightarrow} V \subset X$, $\left(G_{V}, \widetilde{U}\right) \rightarrow U \subset Y, U=V \cap Y$, if $\widetilde{y} \in \widetilde{U}, Z \in N_{\widetilde{U} / \widetilde{V}, \mathbf{R}}$, let $t \in \mathbf{R} \rightarrow x_{t}=$ $\exp _{\widetilde{y}}^{X}(t \widetilde{Z}) \in \widetilde{V}$ be the geodesic in $\widetilde{V}$ which is such that $x_{0}=\widetilde{y}, d x /\left.d t\right|_{t=0}=\widetilde{Z}$. Then for $g \in G_{V}$,

$$
g \exp _{\widetilde{y}}^{X}(t \widetilde{Z})=\exp _{g \widetilde{y}}^{X}(t g \widetilde{Z}) .
$$

So if $(y, Z) \in N_{\mathbf{R}}$ and $|Z|$ is very small, the $\operatorname{map}(y, Z) \in N_{\mathbf{R}} \rightarrow \exp _{y}^{X}(Z) \in X$ is well defined. For $0<\varepsilon<+\infty$, set $B_{\varepsilon}=\left\{Z \in N_{\mathbf{R}} ;|Z|<\varepsilon\right\}$. Since $X$ and $Y$ are compact, there exists $\varepsilon_{0}>0$ such that for $0<\varepsilon<\varepsilon_{0}$, the map $(y, Z) \in N_{\mathbf{R}} \rightarrow$ $\exp _{y}^{X}(Z) \in X$ is a diffeomorphism from $B_{\varepsilon}$ on a tubular neighborhood $\mathcal{U}_{\varepsilon}$ of $Y$ in $X$. From now on, we will identify $B_{\varepsilon}$ with $\mathcal{U}_{\varepsilon}$. Also we will use the notation $x=(y, Z)$ instead of $\exp _{y}^{X}(Z)$. Finally, we identify $y \in Y$ with $(y, 0) \in N_{\mathbf{R}}$. Let $\kappa(y, Z)$ be the smooth positive function defined on $B_{\varepsilon}$ by the equation

$$
d v_{X}(y, Z)=\kappa(y, Z) d v_{Y}(y) d v_{N_{y}}(Z) .
$$

For $y \in Y$, we denote $\widetilde{y} \in \widetilde{V}$ corresponding to $y \in V \subset Y$. Let $\mu(y)$ be the smallest nonzero eigenvalue of the self-adjoint nonnegative operator $V^{2}(\widetilde{y})$. Since $\operatorname{Ker} V$ is a smooth vector bundle on $Y$, the function $y \in Y \rightarrow \mu(y) \in \mathbf{R}_{+}^{*}$ is continuous. Since $Y$ is compact, the function $\mu$ has a positive lower bound $2 b$ on $Y$. We may and will assume that $\varepsilon_{0}>0$ is small enough so that if $x \in \mathcal{U}_{\varepsilon_{0}}, b$ is not an eigenvalue of $V^{2}(\widetilde{x})\left(\widetilde{x} \in \tau_{V}^{-1}(x)\right)$. 
For $0 \leq k \leq m, x \in \mathcal{U}_{\varepsilon_{0}}, \xi_{k, \widetilde{x}}^{-}$(resp. $\xi_{k, \widetilde{x}}^{+}$) denotes the direct sum of the eigenspaces of the restriction of $V^{2}(\widetilde{x})$ to $\xi_{k, \widetilde{x}}$ corresponding to eigenvalues which are smaller (resp. larger) than $b$. Then $\xi_{k, \widetilde{x}}^{-}, \xi_{k, \widetilde{x}}^{+}$define orbifold vector bundles $\xi_{k, x}^{-}, \xi_{k, x}^{+}$on $\mathcal{U}_{\varepsilon_{0}}$.

If $x=(y, Z) \in \mathcal{U}_{\varepsilon_{0}},\left(\Lambda\left(T^{*(0,1)} X\right) \widehat{\otimes} \xi\right)_{x}$ is identified with $\left(\Lambda\left(T^{*(0,1)} X\right) \widehat{\otimes} \xi\right)_{y}$ as in [13, $\S 8 \mathrm{~g})]$, and this identification preserves the metrics and the $\mathbf{Z}$-gradings associated to the operators $\mathrm{N}_{V}^{X}$ and $\mathrm{N}_{H}$.

Let $\mathbf{E}^{ \pm}, \mathbf{E}$ be the set of smooth sections of

$$
\left.\tilde{\pi}^{*}\left(\Lambda\left(T^{*(0,1)} X\right) \widehat{\otimes} \xi^{ \pm}\right)\right|_{Y},\left.\quad \tilde{\pi}^{*}\left(\Lambda\left(T^{*(0,1)} X\right) \widehat{\otimes} \xi\right)\right|_{Y}
$$

on the total space of $N_{\mathbf{R}}$. Let $E^{\mu}$ (resp. $\mathbf{E}^{\mu}, F^{\mu}$ ) be the set of sections of $\Lambda\left(T^{*(0,1)} X\right) \widehat{\otimes} \xi$ (resp. of $\tilde{\pi}^{*}\left(\left.\left(\Lambda\left(T^{*(0,1)} X\right) \widehat{\otimes} \xi\right)\right|_{Y}\right)$ on the total space of $N_{\mathbf{R}}$, of $\Lambda\left(T^{*(0,1)} Y\right) \otimes \eta$ over $\left.Y\right)$, which lie in the $\mu^{\text {th }}$ Sobolev space.

If $y \in Y, Z \in N_{\mathbf{R}, y}$, set

$$
\beta_{y}=\exp \left(\theta_{y}-\frac{|Z|^{2}}{2}\right)
$$

Let $\psi$ be the linear map $\psi: \sigma \in F^{0} \rightarrow \sigma \beta \in \mathbf{E}^{0}$. Let $\mathbf{E}^{\prime}, 0$ be the image of $F^{0}$ by $\psi$ in $\mathbf{E}^{0}$. Then $\mathbf{E}^{\prime, 0} \subset \mathbf{E}^{-, 0}$. Let $p$ be the orthogonal projection operator from $\mathbf{E}^{0}$ on $\mathbf{E}^{\prime}, 0$. One then easily finds that if $s \in \mathbf{E}^{0}$,

$$
p s(y, Z)=\frac{m_{X, Y}}{\pi^{\operatorname{dim} N}} \exp \left(-\frac{|Z|^{2}}{2}\right) q \int_{N_{\mathbf{R}, y}} \exp \left(-\frac{\left|Z^{\prime}\right|^{2}}{2}\right) s\left(y, Z^{\prime}\right) d v_{N}\left(Z^{\prime}\right) .
$$

Here the integral $\int_{N_{\mathbf{R}, y}}=\frac{1}{m_{X, Y}} \int_{\widetilde{N}_{\mathbf{R}, \tilde{y}}}$ is considered in the sense of the integral on orbifolds.

We now take $\left.\varepsilon \in] 0, \varepsilon_{0} / 2\right]$. In the following the constants in our estimates will depend on $\varepsilon$. Let $\gamma$ be a smooth function on $\mathbf{R}$ with values in $[0,1]$ such that $\gamma(a)=1$ for $|a| \leq \frac{1}{2}, \gamma(a)=0$ for $|a| \geq 1$. If $Z \in N_{\mathbf{R}}$, set

$$
\rho(Z)=\gamma\left(\frac{|Z|}{\varepsilon}\right) .
$$

For $T>0, y \in Y$, set

$$
\alpha_{T}(y)=\int_{N_{\mathbf{R}, y}} \exp \left(-T|Z|^{2}\right) \rho^{2}(Z) \frac{d v_{N}(Z)}{(2 \pi)^{\operatorname{dim} N}} .
$$

Clearly, for $1 \leq j \leq d, \alpha_{T}$ takes the constant value $\alpha_{T, j}$ on $Y^{j}$. For $\mu \geq 0, T>0$, let $I_{T}$ be the linear map

$$
\sigma \in F^{\mu} \rightarrow I_{T} \sigma(y, Z)=\left(\alpha_{T} 2^{\operatorname{dim} N}\right)^{-1 / 2} \rho(Z) \exp \left(\theta-\frac{T|Z|^{2}}{2}\right) \sigma \in \mathbf{E}^{\mu} .
$$

Set $J_{T}=\kappa^{-1 / 2} I_{T}$. For $\mu \geq 0, T>0$, let $\mathbf{E}_{T}^{\mu}\left(\right.$ resp. $\left.E_{T}^{\mu}\right)$ be the image of $F^{\mu}$ in $\mathbf{E}^{\mu}$ by $I_{T}$ (resp. $E^{\mu}$ by $J_{T}$ ). Let $p_{T}$ be the orthogonal projection operators from $\mathbf{E}^{0}$ on $\mathbf{E}_{T}^{0}$. 
Proposition 6.10. If $s \in \mathbf{E}^{0}$, and if $y \in Y, Z \in N_{\mathbf{R}, y}$, then

$$
\begin{aligned}
\left(p_{T} s\right)(y, Z)=\frac{\rho(Z)}{\alpha_{T}} \exp ( & \left.-\frac{T|Z|^{2}}{2}\right) \\
& \times q \int_{N_{\mathbf{R}, y}} \rho\left(Z^{\prime}\right) \exp \left(-\frac{T\left|Z^{\prime}\right|^{2}}{2}\right) s\left(y, Z^{\prime}\right) \frac{d v_{N}\left(Z^{\prime}\right)}{(2 \pi)^{\operatorname{dim} N}} .
\end{aligned}
$$

Proof. The proof is elementary and is left to the reader.

Thus we have extended all constructions of [13, $\S 8,9]$ in our case. By proceeding as in [13, $\S 8,9]$, we get Theorems 6.3, 6.4.

6.5. Proof of Theorem 6.8. We use the notation in [13, $\S 10]$. Let $\operatorname{Sp}\left(D^{Y}\right), \operatorname{Sp}\left(A_{T}\right)$ be the spectrum of $D^{Y}, A_{T}$. Let $\left.\left.c_{2} \in\right] 0,1\right]$ be a constant fixed once and for all such that

$$
\operatorname{Sp}\left(D^{Y}\right) \cap\left\{\lambda \in \mathbf{R},|\lambda| \leq 2 c_{2}\right\} \subset\{0\} .
$$

Theorem 6.11. There exists $T_{0} \geq 1$ such that for $T \geq T_{0}$,

$$
\operatorname{Sp}\left(A_{T}\right) \cap\left\{|\lambda| \leq c_{2}\right\} \subset\{0\} .
$$

Proof. By using Theorem 3.4 and proceeding as in the proof of [13, Theorem 9.25], we have Theorem 6.11.

As in Section 6.4, we identify $B_{j, \varepsilon_{0} / 2}=\left\{Z \in N_{\mathbf{R}, j} ;|Z|<\varepsilon_{0} / 2\right\}$ with a tubular neighborhood $\mathcal{U}_{j, \varepsilon_{0} / 2}$ of $Y^{j}$ in $X$. Since $\mathcal{U}_{\varepsilon_{0}}$ is a tubular neighborhood of $Y=$ $\bigcup_{j=1}^{d} Y^{j}$ in $X$, we deduce that if $j \neq j^{\prime}$, then $\overline{\mathcal{U}}_{j, \varepsilon_{0} / 2} \cap \overline{\mathcal{U}}_{j^{\prime}, \varepsilon_{0} / 2}=\emptyset$.

Let $Q$ be the orthogonal projection operator from $F^{0}$ on $\operatorname{Ker}\left(D^{Y}\right)$. We fix $\left.\varepsilon \in] 0, \varepsilon_{0} / 4\right]$.

To prove Theorem 6.8, from the proof of [13, Theorem 10.1], the crucial point is to show that for any $\sigma \in \operatorname{Ker}\left(D^{Y^{j}}\right), \lambda \in \mathbf{C},|\lambda|=c_{2} / 2, m \in \mathbf{N}$, there exists $s_{m}(\lambda, T) \in E^{1}$ such that (cf. [13, (10.8)])

$$
\begin{aligned}
& s_{m}(\lambda, T)=0 \quad \text { on } \quad X \backslash \overline{\mathcal{U}}_{j, \varepsilon_{0} / 2}, \\
& \left\|\left(\lambda-A_{T}\right) s_{m}(\lambda, T)-J_{T} \sigma\right\|=O\left(T^{-m}\right) .
\end{aligned}
$$

To prove (6.27), we will treat in the local card $\left(G_{V}, \widetilde{V}\right) \rightarrow V \subset X$. We easily verify that on $\widetilde{V}$, the construction of $s_{m}(\lambda, T)$ in [13, (10.9)-(10.15)] is $G_{V}$-equivariant. Thus we have constructed $s_{m}(\lambda, T)$ in our case.

By proceeding as in [13, §10], we have Theorem 6.8

\subsection{Proof of Theorem 6.5.}

Proposition 6.12. Let $T_{0} \in[0,+\infty[$. There exists $C>0$ such that for $u \in$ ] $0,1], T \in\left[0, T_{0}\right]$,

$$
\begin{aligned}
& \mid \operatorname{Tr}_{s}[\left.\mathrm{N}_{H} \exp \left(-\left(u D^{X}+T V\right)^{2}\right)\right] \\
& \quad-\sum_{i} \frac{1}{m_{i, X}} \int_{X_{i}} \operatorname{Td}^{\Sigma}\left(T X, g^{T X}\right) \Phi \operatorname{Tr}_{s}^{\Sigma}\left[\mathrm{N}_{H} \exp \left(-C_{T^{2}}^{2}\right)\right] \mid \leq C u \\
&\left|\operatorname{Tr}_{s}\left[\mathrm{~N}_{H} \exp \left(-\left(u D^{X}+T V\right)^{2}\right)\right]-\operatorname{Tr}_{s}\left[\mathrm{~N}_{H} \exp \left(-u^{2} D^{X, 2}\right)\right]\right| \leq C T .
\end{aligned}
$$


Proof. By proceeding as in [13, Proof of Proposition 11.1] and Section 2.3] we easily find that for $T \geq 0$, as $u \rightarrow 0$

$$
\begin{aligned}
& \operatorname{Tr}_{s}\left[\mathrm{~N}_{H} \exp \left(-\left(u D^{X}+T V\right)^{2}\right)\right] \\
&=\sum_{i} \frac{1}{m_{i, X}} \int_{X_{i}} \operatorname{Td}^{\Sigma}\left(T X, g^{T X}\right) \Phi \operatorname{Tr}_{s}^{\Sigma}\left[\mathrm{N}_{H} \exp \left(-C_{T^{2}}^{2}\right)\right]+O(u) .
\end{aligned}
$$

Since $T$ only plays the role of a parameter, one obtains the existence of $C>0$ such that the first inequality in (6.28) holds. By [13, (11.13)] and the technique of Section 2 one finds that the second inequality in (6.28) follows.

In the following, $\alpha$ is a positive constant and the precise value of $\alpha$ should be determinate as in [13, §13e)]. Let $f$ be a smooth even function defined on $\mathbf{R}$ with values in $[0,1]$, such that $f(a)=1$ for $|a| \leq \alpha / 2$, and $f(a)=0$ for $|a| \geq \alpha$. Set $g(t)=1-f(t)$.

Definition 6.13. For $u \in] 0,1], a \in \mathbf{C}$, set

$$
\begin{aligned}
& F_{u}(a)=\int_{-\infty}^{+\infty} \exp (i t a \sqrt{2}) \exp \left(\frac{-t^{2}}{2}\right) f(u t) \frac{d t}{\sqrt{2 \pi}}, \\
& G_{u}(a)=\int_{-\infty}^{+\infty} \exp (i t a \sqrt{2}) \exp \left(\frac{-t^{2}}{2}\right) g(u t) \frac{d t}{\sqrt{2 \pi}}, \\
& H_{u}(a)=\int_{-\infty}^{+\infty} \exp (i t a \sqrt{2}) \exp \left(\frac{-t^{2}}{2 u^{2}}\right) g(t) \frac{d t}{u \sqrt{2 \pi}} .
\end{aligned}
$$

Clearly

$$
\begin{aligned}
& G_{u}(a)=H_{u}\left(\frac{a}{u}\right), \\
& F_{u}\left(u D^{X}+u T V\right)+G_{u}\left(u D^{X}+u T V\right)=\exp \left(-u^{2}\left(D^{X}+T V\right)^{2}\right) .
\end{aligned}
$$

Theorem 6.14. There exist $c>0, C>0$ such that for $u \in] 0,1], T \geq 0$,

$$
\left|\operatorname{Tr}_{s}\left[\mathrm{~N}_{H} G_{u}\left(u D^{X}+u T V\right)\right]\right| \leq c \exp \left(\frac{-C}{u^{2}}\right) \text {. }
$$

Proof. Let $\Delta^{\prime}$ be the contour in C: From $+\infty+i$ to $-1+i$, then from $-1+i$ to $-1-i$, and then from $-1-i$ to $+\infty-i$.

Let $\widetilde{H}_{u, p}(p \in \mathbf{N})$ be the function defined in [13, (11.22)] which verifies that for any $m \in \mathbf{N}$, there exist $c_{m}, C_{m}>0$ such that $\sup _{a \in \Delta^{\prime}}|a|^{m}\left|\widetilde{H}_{u, p}(a)\right| \leq c_{m} \exp \left(-\frac{C_{m}}{u^{2}}\right)$, and

$$
H_{u}\left(A_{T}\right)=\frac{1}{2 \pi i} \int_{\Delta^{\prime}} \frac{\widetilde{H}_{u, p}(\lambda)}{\left(\lambda-A_{T}^{2}\right)^{p}} d \lambda .
$$

Now in our case, [7, Theorems 9.14, 9.17] still holds. Then by proceeding as in the proof of [13, (9.114)], we get [6.32).

From the proof of the finite propagation speed of solutions of hyperbolic equations on smooth manifolds [31, I $\S 2.8]$, we know the proof can be extended to orbifolds. Thus the wave operator $\exp \left(i t \sqrt{2}\left|u D^{X}+u T V\right|\right)$ is well defined on $X$. Now by (6.30),

$$
F_{u}\left(u D^{X}+u T V\right)=\int_{-\alpha / u}^{\alpha / u} \exp \left(i t \sqrt{2}\left|u D^{X}+u T V\right|\right) \exp \left(\frac{-t^{2}}{2}\right) f(u t) \frac{d t}{\sqrt{2 \pi}} .
$$


Let $F_{u}\left(u D^{X}+u T V\right)\left(x, x^{\prime}\right)\left(x, x^{\prime} \in X\right)$ be the smooth kernel of $F_{u}\left(u D^{X}+u T V\right)$ with respect to $d v_{X}\left(x^{\prime}\right) /(2 \pi)^{\operatorname{dim} X}$. Then

$$
\operatorname{Tr}_{s}\left[\mathrm{~N}_{H} F_{u}\left(u D^{X}+u T V\right)\right]=\int_{X} \operatorname{Tr}_{s}\left[\mathrm{~N}_{H} F_{u}\left(u D^{X}+u T V\right)(x, x)\right] \frac{d v_{X}(x)}{(2 \pi)^{\operatorname{dim} X}} .
$$

Using (6.34), we see that if $x \in X, F_{u}\left(u D^{X}+u T V\right)\left(x, x^{\prime}\right)$ vanishes for $d^{X}\left(x, x^{\prime}\right)>\alpha$ and only depends on the restriction of $D^{X}+T V$ to $B^{X}(x, \alpha)$. By Theorem 6.14 we find that the proof of Theorem 6.5 has been reduced to a local problem on $X$.

Let $\left(G_{x_{i}}, \widetilde{V}_{x_{i}}\right)_{i \in I}$ (here $\widetilde{V}_{x}$ is a neighborhood of $0 \in \mathbf{C}^{m}$ and $G_{x}$ acts linearly on $\left.\mathbf{C}^{m}\right)$ be a covering of $X$ such that $\left(G_{x_{i}}, \frac{1}{2} \widetilde{V}_{x_{i}}\right)_{i \in I}$ is also a covering of $X$. Let $\rho_{i}$ be a partition of unity subordinate to $\left(G_{x_{i}}, \frac{1}{2} \widetilde{V}_{x_{i}}\right)_{i \in I}$, the covering of $X$. Let $k(\widetilde{x})$ be such that for $|\widetilde{x}| \leq \frac{\varepsilon}{2}$,

$$
d v_{\widetilde{V}_{x_{i}}}(\widetilde{x})=k(\widetilde{x}) d v_{T_{\widetilde{x}_{i}} \widetilde{V}_{x_{i}}} .
$$

We replace $X$ by $T \tilde{X} / G_{x_{i}}=\mathbf{C}^{n} / G_{x_{i}}(n=\operatorname{dim} X)$, such that $0 \in(T \widetilde{X})_{x_{i}}$ representing $\widetilde{x_{i}}$, and that the extended fibration over $\mathbf{C}^{n}$ coincides with the given fibration over $B(0, \varepsilon) \subset \mathbf{C}^{n}$.

Let

$$
F_{u}\left(u D^{X}+u T V\right)\left(\widetilde{x}, \widetilde{x}^{\prime}\right) \quad\left(\widetilde{x}, \widetilde{x}^{\prime} \in T \widetilde{V}_{x_{i}}\right)
$$

be the smooth kernel of $F_{u}\left(u D^{X}+u T V\right)$ with respect to $d v_{T \widetilde{V}_{x_{i}}}\left(\widetilde{x}^{\prime}\right) /(2 \pi)^{\operatorname{dim} X}$. By (2.2) and the above discussion, for $x \in B\left(x_{i}, \frac{\varepsilon}{2}\right)$, we get

$$
F_{u}\left(u D^{X}+u T V\right)(x, x)=\frac{1}{\left|K_{U}^{\xi}\right|} \sum_{g \in G_{x_{i}}^{\xi}} g F_{u}\left(u D^{X}+u T V\right)\left(g^{-1} \widetilde{x}, \widetilde{x}\right) k(\widetilde{x}) .
$$

By [6. Theorem 11.7], Section 4 (6.35), 6.37), we get Theorem 6.5.

6.7. Proof of Theorem 6.6. By 6.31), 6.32), we know that to establish Theorem 6.6. we just need to show that as $u \rightarrow 0$,

$$
\begin{aligned}
& \operatorname{Tr}_{s}\left[\mathrm{~N}_{H} F_{u}\left(u D^{X}+\frac{T}{u} V\right)\right] \\
&=\sum_{i, j ; Y_{j}^{\prime} \subset X_{i}} \frac{1}{m_{i, X} m_{X_{i}, Y_{j}^{\prime}}} \int_{Y_{j}^{\prime}} \Phi \operatorname{Tr}_{s}^{\Sigma}\left[\mathrm{N}_{H} \exp \left(-\mathcal{B}_{T^{2}}^{2}\right)\right] \operatorname{ch}^{\Sigma}\left(\eta, h^{\eta}\right) .
\end{aligned}
$$

By the argument in Section 6.5 the proof of Theorem 6.6 has been reduced to a local problem on $X$, and we replace $X$ by $T \tilde{X} / G_{x_{i}}=\mathbf{C}^{n} / G_{x_{i}}$ as in Section 6.6 By (6.37), for $x \in B\left(x_{i}, \frac{\varepsilon}{2}\right)$, we get

$$
F_{u}\left(D^{X}+\frac{T}{u} V\right)(x, x)=\frac{1}{\left|K_{U}^{\xi}\right|} \sum_{g \in G_{x_{i}}^{\xi}} g F_{u}\left(D^{X}+\frac{T}{u} V\right)\left(g^{-1} \widetilde{x}, \widetilde{x}\right) k(\widetilde{x}) .
$$

By [6. Theorem 12.3], (4.4), (6.35), (6.39), we get (6.11).

6.8. Proof of Theorem 6.7. We use the notation of Section 6.5

Theorem 6.15. There exist $c>0, C>0$, such that for $u \in] 0,1], T \geq 1$,

$$
\left|\operatorname{Tr}_{s}\left[\mathrm{~N}_{H} G_{u}\left(u D^{X}+\frac{T}{u} V\right)\right]-\frac{1}{2} \operatorname{dim} N \chi(\eta) G_{u}(0)\right| \leq \frac{c}{\sqrt{T}} \exp \left(\frac{-C}{u^{2}}\right) .
$$


Proof. The proof of our theorem is essentially the same as the proof of 13, Theorem 13.4], [6] Theorem 13.4]. Of course, we use the argument of Section [6.5. In fact, we find that

$$
\left|\operatorname{Tr}_{s}\left[\mathrm{~N}_{H} G_{u}\left(u D^{X}+\frac{T}{u} V\right)\right]-\operatorname{Tr}_{s}\left[N_{H}^{\theta} G_{u}\left(u D^{Y}\right)\right]\right| \leq \frac{c u}{\sqrt{T}} \exp \left(\frac{-C}{u^{2}}\right) .
$$

By [13, Proposition 8.4], $N_{H}^{\theta}=\frac{1}{2} \operatorname{dim} N$. Recall that $G_{u}(a)$ is a holomorphic function of $a^{2}$. By an analogue of the Mckean-Singer formula, we find that for $1 \leq j \leq d$

$$
\operatorname{Tr}_{s}\left[G_{u}\left(u D_{j}^{Y}\right)\right]=\chi\left(\eta_{j}\right) G_{u}(0) .
$$

Using (6.41), (6.42), we get (6.40).

In view of Theorem 6.7 and (6.31), we see that to prove Theorem 6.7, we only need to show there exist $C>0, \delta \in] 0,1]$ such that for $u \in] 0,1], T \geq 1$,

$$
\left|\operatorname{Tr}_{s}\left[\mathrm{~N}_{H} F_{u}\left(u D^{X}+\frac{T}{u} V\right)\right]-\frac{1}{2} \operatorname{dim} N \chi(\eta) F_{u}(0)\right| \leq \frac{C}{T^{\delta}} .
$$

As in Section 6.5, we replace $X$ by $T \tilde{X} / G_{x_{i}}=\mathbf{C}^{n} / G_{x_{i}}(n=\operatorname{dim} X)$. By [6. Theorem 13.6, Remark 13.7], (6.35) and (6.39), we have (6.43).

By (6.41), (6.43), we get Theorem 6.7.

\section{ACKNOWLEDGMENTS}

We are very much indebted to Professor Jean-Michel Bismut for very helpful discussions and suggestions. We also thank the referee for his careful reading of the manuscript.

\section{REFERENCES}

[1] Adem A., Morava J. and Ruan Y. (Eds), Orbifolds in mathematics and physics. Contemporary Mathematics 310 (2002). MR1950939 (2003g:00020)

[2] Berline N., Getzler E., and Vergne M., Heat kernels and Dirac operators, Grundl. Math. Wiss. 298, Springer, Berlin-Heidelberg-New York 1992. MR.1215720 (94e:58130)

[3] Bismut J.-M., Superconnection currents and complex immersions, Invent. Math. 99 (1990), 59-113. MR1029391 (91b:58240)

[4] Bismut J.-M., Koszul complexes, harmonic oscillators and the Todd class, J.A.M.S. 3 (1990), 159-256. MF,1017783 (91b:58245)

[5] Bismut J.-M., Equivariant short exact sequences of vector bundles and their analytic torsion forms. Comp. Math. 93 (1994), 291-354. MF1300765 (96g:58201)

[6] Bismut J.-M., Equivariant immersions and Quillen metrics, J. Diff. Geom. 41 (1995), 53-159. MR 1316553 (96m:58261)

[7] Bismut J.-M., Families of immersions, and higher analytic torsion, Astérisque 244, 1997. MR:1623496 (2000b:58057)

[8] Bismut J.-M., Gillet H., Soulé C., Analytic torsion and holomorphic determinant bundles. I, Comm. Math. Phys. 115 (1988), 49-78. MR0929146 (89g:58192a)

[9] Bismut J.-M., Gillet H., Soulé C., Analytic torsion and holomorphic determinant bundles. II, Comm. Math. Phys. 115 (1988), 79-126. MR0929146 (89g:58192a)

[10] Bismut J.-M., Gillet H., Soulé C., Analytic torsion and holomorphic determinant bundles. III, Comm. Math. Phys. 115 (1988), 301-351. MR0931666 (89g:58192c)

[11] Bismut J.-M., Gillet H., Soulé C., Bott-Chern currents and complex immersions, Duke Math. Journal 60 (1990), 255-284. MR1047123 (91d:58239)

[12] Bismut J.-M., Köhler K., Higher analytic torsion forms for direct images and anomaly formulas. J. of. Alg. Geom. 1 (1992), 647-684. MR1174905 (94a:58209)

[13] Bismut J.-M. and Lebeau G., Complex immersions and Quillen metrics. Publ. Math. IHES., Vol. 74, 1991, 1-297. MR1188532(94a:58205) 
[14] Bismut J.-M. and Ma X., Holomorphic immersions and equivariant torsion forms, J. Reine Angew. Math. 575 (2004), 189-235. MR2097553

[15] Cartan H., Quotient d'un espace analytique par un groupe d'automorphismes. Algebraic Geometry and Topology. Fox R.H., Spencer D.C., and Tucker A.W. eds. Princeton Univ Press, Princeton 1957, 90-102. MR0084174 (18:823b)

[16] Cartan H., and Eilenberg S., Homological Algebra, Princeton 1956. MR0077480 (17:1040e)

[17] Grothendieck A., Sur quelques points d'algèbre homologique, Tôhoku Math. J. 9 (1957), 119-221. MF 0102537(21:1328)

[18] Gillet H., Soulé C., Analytic torsion and the arithmetic Todd genus, Topology 30 (1991), 21-54. MR 1081932 (92d:14015)

[19] Gillet H., Soulé C., An arithmetic Riemann-Roch Theorem, Invent. Math. 110 (1992), 473543. MR 1189489 (94f:14019)

[20] Kawasaki T., The Signature theorem for V-manifolds. Topology 17 (1978), 75-83. MR0474432 $(57: 14072)$

[21] Kawasaki T., The Riemann-Roch theorem for V-manifolds. Osaka J. Math. 16 (1979), 151159. MR0527023 (80f:58042)

[22] Kawasaki T., The Index of elliptic operators for V-manifolds. Nagoya. Math. J. 84 (1981), 135-157. MF0641150 (83i:58095)

[23] Knudsen P.F., Mumford D., The projectivity of the moduli space of stable curves, I, Preliminaires on "det" and "div", Math. Scand. 39 (1976), 19-55. MR0437541 (55:10465)

[24] Köhler K., Roessler D., A fixed point formula of Lefschetz type in Arakelov geometry. I. Statement and proof. Invent. Math. 145 (2001), 333-396. MR1872550 (2003m:14041a)

[25] Lawson H.B., Michelson M.L., Spin geometry, Princeton Univ Press, Princeton 1989. MR:1031992 (91g:53001)

[26] Ma X., Formes de torsion analytique et familles de submersions. I, Bull. Soc. Math. France 127 (1999), 541-621; II, Asian J. of Math. 4 (2000), 633-668. MR.1765553 (2001j:58059). MR $1796698(2002 \mathrm{e}: 58066)$

[27] Ma X., Submersions and equivariant Quillen metrics, Ann. Inst. Fourier 50 (2000), 15391588. MR.1800127 (2002c:58053)

[28] Quillen D., Determinants of Cauchy-Riemann operators over a Riemann surface, Funct. Anal. Appl. 19 (1985), 31-34. MR0783704 (86g:32035)

[29] Ray D.B., Singer I.M., Analytic torsion for complex manifolds, Ann. of Math. 98 (1973), 154-177. MF0383463 (52:4344)

[30] Satake I., The Gauss-Bonnet theorem for V-manifolds, J. Math. Soc. Japon. 9 (1957), 464492. MR0095520 (20:2022)

[31] Taylor M., Partial Differential Equations I, II. Applied Mathematical Sciences 115, 116, Springer 1996. MR:1395148|(98b:35002b)] MR1395149 (98b:35003)

Centre de Mathématiques, UMR 7640 du CNRS, École Polytechnique, 91128 Palaiseau Cedex, France

E-mail address: ma@math.polytechnique.fr 